\title{
Argaric Sociology: Sex and Death
}

\author{
Sociología argárica: sexo y muerte
}

\author{
Vicente LulL*, Rafael Micó ${ }^{* *}$, Cristina Rihuete HerradA***, Roberto RisCH**** \\ * Department of Prehistory, Autonomous University of Barcelona \\ vicenc.lull@uab.cat \\ ** Department of Prehistory, Autonomous University of Barcelona \\ rafael.mico@uab.cat \\ *** Department of Prehistory, Autonomous University of Barcelona \\ cristina.rihuete@uab.cat \\ **** Department of Prehistory, Autonomous University of Barcelona \\ robert.risch@uab.cat
}

Recibido: 11-04-2016

Aceptado: 14-07-2016

\begin{abstract}
The detailed, rich and diverse Argaric funerary record offers an opportunity to explore social dimensions that usually remain elusive for prehistoric research, such us social rules on kinship rights and obligations, sexual tolerance and the role of funerary practices in preserving the economic and political organization. This paper addresses these topics through an analysis of the social meaning of Argaric double tombs by looking at body treatment and composition of grave goods assemblages according to gender and class affiliation. The Argaric seems to have been a conservative society, scarcely tolerant regarding homosexuality, and willing to celebrate ancestry associated to certain places as a means of asserting residence and property rights.
\end{abstract}

KeYwords: Burial, El Argar, Double Tombs, Social Organization.

\section{RESUMEN}

La variedad, abundancia y detalle del registro funerario argárico permite explorar aspectos por lo general vedados a la investigación de las sociedades prehistóricas. Las normas sociales sobre la distribución de obligaciones y derechos según el parentesco, la permisividad respecto a las relaciones sexuales y el papel de las prácticas funerarias en el mantenimiento de la organización económico-politica son algunos de dichos aspectos. En este artículo se propone un acercamiento a los mismos mediante un análisis del significado social de las tumbas dobles argáricas, la composición de los ajuares según el sexo y la clase social del individuo inhumado, así como el tratamiento y disposición de los cadáveres. Las conclusiones dibujan una sociedad conservadora, poco tolerante respecto la homosexualidad y que celebra positivamente los vínculos genealógicos asociados a ciertos espacios, afirmando así derechos de permanencia y propiedad.

Palabras Clave: Enterramientos, El Argar, tumbas dobles, organización social.

SumARIO: 1 . Sex, age and kinship in the Argaric society based on double burials. 2. The archaeological visibility of women and men. 3. Sex and transgression. 4. Conclusions. 
Societies can be assessed in terms of the relations maintained by their members. A tolerant society attempts to harmonise behaviours to enable fluid and open relations. A repressive society seeks to preserve a specific lifestyle, which it imposes by means of norms that decide what is fair and what is unfair, good or bad, according to the interests of its rulers.

In combination or separately, sexual relations, death and violence can be used as yardsticks to assess social relations:

- If the sexual relations are permissive, with shared enjoyment as both its means and end, then it would only be paradoxical if they were sources of interpersonal violence. Conversely, if they are constrained and exclusive, they end up resembling a relation of ownership, whose maintenance is based on fear, and which often is ended by murder.

- $\quad$ Societies deal with death in different ways. Funerary practices may indicate whether a given society established public or private ceremonies and, for each case, the degree of participation and involvement of individuals and groups.

- To speak of "violence" involves more than noticing an aggression. ${ }^{2}$ The notion alludes to a state of social-political relations in which conflict has made its home, and in which the categories "allies" and "enemies" have been established.

In this article we will focus on the first two factors, sex and death, as these are strongly interwoven within the archaeological investigation of Argaric society. We leave for another occasion the treatment of the forms of violence, since the analysis required involves new empirical findings that need yet to be investigated.

\section{Sex, age and kinship in the Argaric society based on double burials}

Argaric society developed between $c a .2200$ and $1550 \mathrm{cal} \mathrm{BCE}$ in south-eastern Iberia. Here we will not dwell on its archaeological features, the achievements and goals of modern research, or the reasons that make it a key reference for the understanding of Later European Prehistory. ${ }^{3}$ Nevertheless, the plentiful and varied funer- ary contexts will be our starting point thanks to unusual confluence of two circumstances:

1. The burials, mostly single or double, materialise forms of funerary treatment to individuals of any age and gender, that we are able to document in detail.

2. The burials were installed in the subsoil of the settlements, which allows to make correspondences between the funerary practices and other areas of social life.

It is usually accepted that not all of the population received the funerary treatment that is attested archaeologically. ${ }^{4}$ In other words, there were social mechanisms that decided which individuals were buried inside settlements and which were not. This bias may condition the scope of the analysis, but at the outset it does not invalidate it, as the demographic profile drawn from the available data fits with that of a entire population. Two pieces of evidence support this claim. First, the data regarding the average age at death from all types of burials indicates a high mortality among infants: $45.6 \%$ (Table 1). This statistic is in line with what is expected in pre-industrial societies and reveals that age was not a factor of exclusion in the intramural burial..$^{5}$ Secondly, the data on mortality and sex suggest significant differences between sites, from some showing an underrepresentation of females (El Oficio), to others reflecting the opposite tendency (Lorca and La Almoloya) (Table 1). As the total sum yields a situation of more or less parity, the variability between sites might be accounted for norms of territorial distribution of the population in which the sexual variable played a relevant role.

Studies of the funerary record have focussed on single burials, since in addition to being the most numerous they provide unequivocal links between grave goods and specific individuals. ${ }^{6}$ Nevertheless, here we will explore the heuristic possibilities presented by double burials, which can enhance the current approaches that draw on the identification of differences in the value of the deposited objects.

It must be pointed out that double interments did not represent an innovation introduced by, or an exclusive feature of, Argaric communities. Double burials are attested in Iberia before 2200 cal BCE, coinciding with the end of the Copper Age and the Bell Beaker phenomenon. Among the double burials dated by radiocarbon, and only 
Table 1. Argaric burials and demographic profile of the lowlands of south-eastern Iberia.

\begin{tabular}{|c|c|c|c|c|c|c|c|c|c|c|c|c|c|c|c|c|}
\hline & \multicolumn{2}{|c|}{ TOTAL } & \multicolumn{2}{|c|}{$\begin{array}{l}\text { El Argar } \\
\text { (Antas, } \\
\text { Almería) }\end{array}$} & \multicolumn{2}{|c|}{$\begin{array}{l}\text { El Oficio } \\
\text { (Cuevas, } \\
\text { Almería) }\end{array}$} & \multicolumn{2}{|c|}{$\begin{array}{c}\text { La Bastida } \\
\text { (Totana, } \\
\text { Murcia) }\end{array}$} & \multicolumn{2}{|c|}{$\begin{array}{c}\text { Fuente } \\
\text { Álamo } \\
\text { (Cuevas, } \\
\text { Almería) }\end{array}$} & \multicolumn{2}{|c|}{$\begin{array}{c}\text { La } \\
\text { Almoloya } \\
\text { (Pliego, } \\
\text { Murcia) }\end{array}$} & \multicolumn{2}{|c|}{$\begin{array}{l}\text { Lorca } \\
\text { (Lorca, } \\
\text { Murcia) }\end{array}$} & \multicolumn{2}{|c|}{$\begin{array}{c}\text { Gatas } \\
\text { (Turre, } \\
\text { Almería) }\end{array}$} \\
\hline & $\mathrm{N}$ & $\%$ & $\mathrm{~N}$ & $\%$ & $\mathrm{~N}$ & $\%$ & $\mathrm{~N}$ & $\%$ & $\mathrm{~N}$ & $\%$ & $\mathrm{~N}$ & $\%$ & $\mathrm{~N}$ & $\%$ & $\mathrm{~N}$ & $\%$ \\
\hline & & & & & & & & & & & & & & & & \\
\hline Burials & 1398 & & 857 & & 170 & & 157 & & 87 & & 53 & & 48 & & 34 & \\
\hline Individual & 1242 & 88.8 & 776 & 90.5 & 156 & 91.8 & 138 & 87.9 & 79 & 90.8 & 42 & 79.2 & 32 & 66.6 & 26 & 76.5 \\
\hline Double & 148 & 10.6 & 80 & 9.3 & 13 & 7.6 & 17 & 10.8 & 8 & 9.2 & 11 & 20.7 & 13 & 27.1 & 7 & 20.6 \\
\hline Triple & 8 & 0.6 & 1 & 0.1 & 1 & 0.6 & 2 & 1.3 & - & - & - & & 3 & 6.2 & 1 & 2.9 \\
\hline Individuals & 1178 & & 588 & & 185 & & 176 & & 65 & & 66 & & 65 & & 42 & \\
\hline Infants & 537 & 45.6 & 256 & 43.6 & 93 & 50.3 & 80 & 45.4 & 30 & 46.1 & 25 & 37.9 & 36 & 55.4 & 21 & 50.0 \\
\hline Juvenile & 64 & 5.4 & 42 & 7.1 & 8 & 4.3 & 9 & 5.1 & 2 & 3.1 & - & & 1 & 1.5 & 2 & 4.8 \\
\hline Adults & 577 & 49.0 & 290 & 49.3 & 84 & 45.4 & 87 & 49.4 & 33 & 50.8 & 41 & 62.1 & 28 & 43.1 & 19 & 45.2 \\
\hline Sex & 391 & & 189 & & 46 & & 36 & & 35 & & 38 & & 30 & & 19 & \\
\hline Male & 192 & 49.1 & 96 & 50.8 & 30 & 65.2 & 17 & 47.2 & 16 & 45.7 & 15 & 39.5 & 11 & 36.6 & 8 & 42.1 \\
\hline Female & 199 & 50.9 & 93 & 49.2 & 16 & 34.8 & 19 & 52.7 & 19 & 54.3 & 23 & 60.5 & 19 & 63.3 & 11 & 57.9 \\
\hline $\begin{array}{l}\text { Double } \\
\text { burials }\end{array}$ & 103 & & 35 & & 13 & & 17 & & 8 & & 11 & & 13 & & 7 & \\
\hline Infant + Infant & 11 & 10.7 & 2 & 5.7 & - & & 3 & 17.6 & - & & 1 & 9.1 & 4 & 30.8 & 1 & 14.3 \\
\hline $\begin{array}{l}\text { Juvenile }+ \\
\text { Juvenile }\end{array}$ & 1 & 1.0 & - & & - & & 1 & 5.9 & - & & - & & - & & - & \\
\hline Adult + Adult & 60 & 58.2 & 16 & 45.7 & 5 & 38.5 & 11 & 64.7 & 7 & 87.5 & 9 & 81.8 & 7 & 53.8 & 6 & 85.7 \\
\hline $\begin{array}{l}\text { Infant }+ \\
\text { Juvenile } \\
\end{array}$ & 1 & 1.0 & - & & - & & - & & - & & - & & 1 & 7.7 & - & \\
\hline $\begin{array}{l}\text { Juvenile + } \\
\text { Adult }\end{array}$ & 4 & 3.9 & 4 & 11.4 & - & & - & & - & & - & & - & & - & \\
\hline Adult + Infant & 26 & 25.2 & 13 & 37.1 & 8 & 61.5 & 2 & 11.8 & 1 & 12.5 & 1 & 9.1 & 1 & 7.7 & - & \\
\hline Bibliography & & & & $2,3,4$ & & $2,3,4$ & $1,3,4$ & $\begin{array}{l}5,6 \\
8,9\end{array}$ & & $\begin{array}{l}3,4 \\
10,11\end{array}$ & & 9,27 & & $\begin{array}{l}3,14 \\
6,17 \\
9,29 \\
1,22\end{array}$ & & $\begin{array}{l}4,23, \\
25,26\end{array}$ \\
\hline
\end{tabular}

\section{Caption:}

Burials: Comprises all cases with data on the number of interred individuals; cenotaphs and looted burials are excluded. The only two burials that in all likelihood contained pregnant women (El Argar 315 and 828) are counted as individual burials.

Individuals: Comprises only those cases for which there is osteological (biological age) data: Infants $=<12$ years; Juveniles $=12-20$ years; Adults $=>20$ years.

Sex: Only the cases in which the sex could be determined osteologically are included.

Bibliography: 1: Siret and Siret 1890, 2: Jacques 1890; 3: Diaries of Pedro Flores (available for direct consult in the Museo Arqueológico Nacional in Madrid; the relevant text is collected in Schubart and Ulreich 1991) 4: Kunter 1990; 5: Inchaurrandieta 1870; 6: Martínez Santa-Olalla et alii 1947; 7: Ruiz Argilés and Posac 1956; 8: Lull et alii 2012; 9: unpublished documentation; 10: Schubart et alii 2000; 11: Schubart 2012; 12: Martínez 1990; 13: Martínez 1995; 14: Martínez et alii 1996; 15: Sánchez Pravía 2001; 16: Martínez and Ponce 2002a; 17: Martínez and Ponce 2002b; 18: Pujante 2003; 19: Gallardo and González 2006; 20: Pérez Richard 2007; 21: Pujante and Martínez 2010; 22: Rihuete et alii 2011; 23: Castro et alii 1994 24: Castro et alii 1995; 25: Castro et alii 2001; 26: Castro et alii 2004; 27: Cuadrado 1945.

as an example, we have the last phase of the funerary complex of La Sima (Soria) ${ }^{7}$, Humanejos (Madrid), ${ }^{8}$ El Castillejo (Toledo) ${ }^{9}$ and Cave 5 of Valle de las Higueras (Toledo) ${ }^{10}$.

From 2200 cal BCE onward in Southern Iberia, the cases dated by radiocarbon become more frequent (e.g. Molinos de Papel ${ }^{11}$-Murcia-, Castillejo del Bonete ${ }^{12}$-Ciudad Real-, Les Raboses ${ }^{13}$ -Valencia-, Camino de las Yeseras ${ }^{14}$-Madrid-, Las Cruces/SE-K ${ }^{15}$-Sevilla-, Torre Velha $3^{16}$, Outeiro Alto $2-$ Beja- $^{17}$ ). The social significance of the examples prior to and contemporary with the 
Table 2. Argaric double burials with data on the age of the individuals interred.

\begin{tabular}{|c|c|c|c|c|c|c|c|c|c|}
\hline Site & Province & $N$ & Ad+Ad & Ad+Juv & Juv+Juv & Ad+Inf & Inf+Inf & Burials & Bibliography \\
\hline $\begin{array}{l}\text { Illeta dels } \\
\text { Banyets }\end{array}$ & Alicante & 4 & 4 & - & - & - & - & $1,2,4$ and 9 & López Padilla et alii 2009 \\
\hline Cabezo Pardo & Alicante & 1 & 1 & & & & & 1 & $\begin{array}{l}\text { López Padilla 2009: 158- } \\
159\end{array}$ \\
\hline El Argar & Almería & 35 & 16 & 4 & - & 13 & 2 & $\begin{array}{l}18,21,86,147, \\
153,155,188, \\
244,245,305, \\
312,356,368, \\
372,406,499, \\
510,609,618, \\
627,644,663, \\
718,738,742, \\
755,757,797, \\
824,880,892, \\
948,977,981 \\
\text { and } 984\end{array}$ & $\begin{array}{l}\text { Siret and Siret 1890; } \\
\text { Jacques 1890; field notes } \\
\text { of Pedro Flores (Museo } \\
\text { Arqueológico Nacional } \\
\text { in Madrid, and Schubart } \\
\text { and Ulreich 1991); Kunter } \\
1990\end{array}$ \\
\hline El Oficio & Almería & 13 & 5 & - & - & 8 & - & $\begin{array}{l}4,9,16,80, \\
137,139,145, \\
198,222,229, \\
236,242 \text { and } \\
283\end{array}$ & $\begin{array}{l}\text { Siret and Siret 1890; } \\
\text { Jacques 1890; field notes } \\
\text { of Pedro Flores (Museo } \\
\text { Arqueológico Nacional } \\
\text { in Madrid, and Schubart } \\
\text { and Ulreich 1991); Kunter } \\
1990\end{array}$ \\
\hline Fuente Álamo & Almería & 8 & 7 & - & - & 1 & - & $\begin{array}{l}9,23,58,69 \\
75,80,89 \text { and } \\
115\end{array}$ & Schubart 2012 \\
\hline Gatas & Almería & 7 & 6 & - & - & - & 1 & $\begin{array}{l}7,13,18,28 \\
33,35 \text { and } 37\end{array}$ & Castro et alii 1995: tab. 3 \\
\hline $\begin{array}{l}\text { Lugarico } \\
\text { Viejo }\end{array}$ & Almería & 1 & 1 & - & - & - & - & 3 & $\begin{array}{l}\text { Siret and Siret 1890: láms. } \\
\text { 15-16; Kunter 1990 }\end{array}$ \\
\hline $\begin{array}{l}\text { Cerro de la } \\
\text { Encantada }\end{array}$ & $\begin{array}{l}\text { Ciudad } \\
\text { Real }\end{array}$ & 3 & 2 & 1 & - & - & - & 10,17 and 37 & $\begin{array}{l}\text { Romero 1988: } 145 \text { and } \\
\text { tab. 1; Lapuente 2008: } \\
\text { tabs. } 1 \text { and } 6\end{array}$ \\
\hline Castellón Alto & Granada & 8 & 6 & - & - & 1 & 1 & $\begin{array}{l}7,18,26,75 \\
85,91,110 \text { and } \\
121\end{array}$ & $\begin{array}{l}\text { Molina et alii 1986: } 358 ; \\
\text { Contreras et alii 1997: } \\
\text { 127; Molina et alii 2003; } \\
\text { Cámara and Molina 2009: } \\
\text { 173-175 and fig. } 3 \text {; Haro } \\
\text { 2012: } 392 \text {; display panels } \\
\text { at the site. }\end{array}$ \\
\hline $\begin{array}{l}\text { Cerro de la } \\
\text { Encina }\end{array}$ & Granada & 7 & 3 & 2 & & 1 & 1 & $\begin{array}{l}6,11,12,14, \\
16,21 \text { and } 22\end{array}$ & $\begin{array}{l}\text { Jiménez and García } \\
\text { Sánchez 1989-1990; } \\
\text { Aranda and Molina 2005 } \\
\text { and 2006; Aranda et alii } \\
\text { 2008; Sánchez Romero } \\
\text { 2008; Sánchez Romero } \\
\text { and Alarcón } 2012 \\
\end{array}$ \\
\hline $\begin{array}{l}\text { Cerro de la } \\
\text { Virgen }\end{array}$ & Granada & 5 & 2 & - & 1 & 1 & 1 & $\begin{array}{l}4,6,22,29 \\
\text { and } 30\end{array}$ & $\begin{array}{l}\text { Schüle 1967; Cámara and } \\
\text { Molina 2009; Delgado } \\
2013\end{array}$ \\
\hline $\begin{array}{l}\text { Cerro del } \\
\text { Culantrillo } \\
\end{array}$ & Granada & 1 & 1 & - & - & - & - & 3 & García Sánchez 1963 \\
\hline $\begin{array}{l}\text { Cerro San } \\
\text { Cristóbal } \\
\end{array}$ & Granada & 3 & 1 & - & - & 2 & - & $7,12,18$ and 28 & Aranda et alii 2012 \\
\hline $\begin{array}{l}\text { Cuesta del } \\
\text { Negro }\end{array}$ & Granada & 8 & 5 & 2 & - & 1 & - & \begin{tabular}{|l|}
$2,4,6,9,19$ \\
21,29 and 31 \\
\end{tabular} & Cámara 2001: tab. 28 \\
\hline $\begin{array}{l}\text { Fuente } \\
\text { Amarga }\end{array}$ & Granada & 1 & 1 & - & - & - & - & 9 & $\begin{array}{l}\text { Fresneda et alii 1999: 235; } \\
\text { Al-Oumaoui 2009: } 39\end{array}$ \\
\hline
\end{tabular}




\begin{tabular}{|c|c|c|c|c|c|c|c|c|c|}
\hline $\begin{array}{l}\text { Baeza (Cerro } \\
\text { del Alcázar) }\end{array}$ & Jaén & 5 & 4 & - & - & 1 & - & $\begin{array}{l}4,5,7,11 \text { and } \\
12\end{array}$ & $\begin{array}{l}\text { Zafra and Pérez Bareas } \\
\text { 1992: fig. 6; Robledo and } \\
\text { Trancho 2003 } \\
\end{array}$ \\
\hline Peñalosa & Jaén & 5 & 2 & - & - & 3 & - & $\begin{array}{l}1,4,13,18 \\
\text { and } 21\end{array}$ & $\begin{array}{l}\text { Contreras et alii 2001: } \\
\text { tab. 14.7; Alarcón 2010: } \\
\text { 1000; Alarcón 2012: 46- } \\
\text { 47; Sánchez and Alarcón } \\
\text { 2012: 76-77 }\end{array}$ \\
\hline $\begin{array}{l}\text { Úbeda (Eras } \\
\text { del Alcázar) }\end{array}$ & Jaén & 1 & 1 & - & - & - & - & 9 & $\begin{array}{l}\text { Lizcano et alii 2009: 39; } \\
\text { Nocete et alii 2010: tab. } 5 \\
\end{array}$ \\
\hline Bajil & Murcia & 1 & 1 & - & - & - & - & 8 & $\begin{array}{l}\text { Eiroa 1993-1994: 69, lám. } \\
\text { XII and fig. } 9\end{array}$ \\
\hline La Almoloya & Murcia & 11 & 8 & - & - & 2 & 1 & $\begin{array}{l}\text { Cuadrado } \\
\text { series: } 1 ; \\
\text { Autonomous } \\
\text { University } \\
\text { of Barcelona } \\
\text { series: } 15,21 \text {, } \\
22,24,26,27 \text {, } \\
30,38,42 \text { and } \\
44\end{array}$ & $\begin{array}{l}\text { Cuadrado 1945: } 380 ; \\
\text { unpublished (Autonomous } \\
\text { University of Barcelona } \\
2013 \text { and } 2014 \\
\text { excavations) }\end{array}$ \\
\hline La Bastida & Murcia & 17 & 11 & - & 1 & 2 & 3 & $\begin{array}{l}\text { Siret series: } \\
\text { 2; SHP series: } \\
11,35,52 \text {, } \\
76,93,102 ; \\
\text { unpublished } \\
\text { SHP series: } 6 \text {; } \\
\text { Autonomous } \\
\text { University } \\
\text { of Barcelona } \\
\text { series: } 6,12,16 \text {, } \\
18,23,40,61 \text {, } \\
65 \text { and } 71\end{array}$ & \begin{tabular}{|l} 
Siret and Siret 1890; \\
Jacques 1890; diaries \\
of Pedro Flores (Museo \\
Arqueológico Nacional \\
in Madrid, and Schubart \\
and Ulreich 1991); Kunter \\
1990; del Val et alii \\
1947; Lull et alii 2012; \\
unpublished (excavations \\
by Jordá and Evans \\
-1950- and Autonomous \\
University of Barcelona \\
-2009/2012-)
\end{tabular} \\
\hline Lorca & Murcia & 13 & 7 & - & - & 2 & 4 & \begin{tabular}{|l} 
Madres \\
Mercedarias \\
Convent series: \\
2 and 7; Madres \\
Mercedarias \\
Church series: \\
6, 11, 14 and \\
17; C/ Los \\
Tintes series: 2; \\
C/Zapatería ${ }^{\circ}$ \\
11 series: 1 and \\
5; Castle series: \\
1, and E3, E4 \\
and E5 \\
\end{tabular} & $\begin{array}{l}\text { Pujante and Martínez } \\
\text { 2010; Rihuete } \text { et alii 2011; } \\
\text { Martínez and Ponce 2002a } \\
\text { y 2002b; Martínez 1995; } \\
\text { Sánchez Pravía } 2001\end{array}$ \\
\hline Monteagudo & Murcia & 1 & - & - & - & 1 & 0 & $\begin{array}{l}\text { Cuesta San } \\
\text { Cayetano series: } \\
\text { UE245 } \\
\end{array}$ & $\begin{array}{l}\text { Unpublished (excavations } \\
\text { by J. A. Zapata) }\end{array}$ \\
\hline $\begin{array}{l}\text { Puntarrón } \\
\text { Chico }\end{array}$ & Murcia & 2 & 1 & - & - & $1 ?$ & - & Cists 15 and 23 & $\begin{array}{l}\text { García Sandoval 1964: } \\
\text { lám XIV-1 and XXVIII-4 }\end{array}$ \\
\hline $\begin{array}{l}\text { Rincón de } \\
\text { Almendricos }\end{array}$ & Murcia & 1 & 1 & - & - & - & - & Cist 9 & $\begin{array}{l}\text { Ayala 1991: } 116-121 \text { and } \\
\text { figs. } 48-50 .\end{array}$ \\
\hline TOTAL & & 162 & 97 & 9 & 2 & 40 & 14 & & \\
\hline$\%$ & & & $66.7 \%$ & & & $24.7 \%$ & $8.6 \%$ & & \\
\hline
\end{tabular}

\section{Caption:}

Biological age categories: $\boldsymbol{A d}=>20$ years; $\boldsymbol{J u} \boldsymbol{v}=12-20$ years; $\boldsymbol{I n f}=<12$ years; Burials: reference number of the graves considered in this study. 
Argaric ones is not necessarily analogous. In fact, the presence of two skeletons in a grave does not automatically point to a "double burial", if we limit this category to only those archaeological entities, as the Argaric, in which a high number of cases confirms its ritual role. On the contrary, the occurrence of two skeletons in a burial may be simply accidental.

In the lowlands of south-eastern Iberia, where most of the Argaric funerary contexts occur, the proportion of double burials with regard to the total number of interments is around $10 \%$, though it can be over $20 \%$ at some sites (Table 1). ${ }^{18}$ With regard to the double burials of the coastal and pre-littoral areas that supply data on the osteological age of their occupants (Table 1), slightly over half of them included more than two adult individuals. ${ }^{19}$ If adult age as a social category began, as it seems, during adolescence, ${ }^{20}$ then the proportion of double burials with two adults in the whole Argaric territory reaches two-thirds (Table 2). On the other hand, double burials of infants represent only $8 \%$ of the cases and those with an adult along with a child $25 \%$.

The picture would have been very different if it had been possible for a double burial to include individuals of any age or sex. The first piece of evidence against the possibility of random combinations stems from the fact that the infants were in all cases interred after the adult one. That is to say, it was possible to add a second individual of an age similar to, or lower than, that of the first occupant, but not vice-versa: an infant never inaugurated a burial that would later accommodate an adult. Put differently, given that the size of the funerary container correlates with the size of the corpse, the age of the first occupant could limit that of the second. ${ }^{21}$

If we class the Argaric population in three age groups, we arrive at a series of combinations (Fig. 1). As the mortality rate in each of the three groups differs (the probability of dying at the age of 12-20-juvenile group-or after 20 years of age-adult group-is not the same as with the first 12 years of life-infant group), the testing of the random deposition of two bodies ought to take into account these six possible combinations, according to the demographic profile of each necropolis. Before we deal with these calculations, we should have a rough idea of what the expected values would be. Since in the whole Argaric world there is approximately the same number of infant and adult burials, we should expect to find the same amount of double burials with

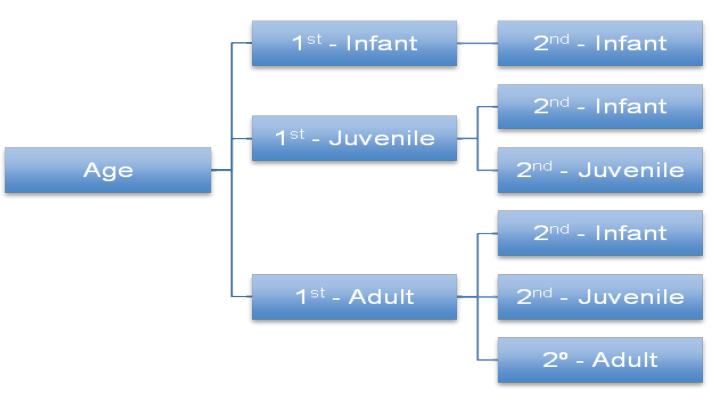

Figure 1. Possible combinations of individuals in burials.

two infants $(\mathrm{I}+\mathrm{I})$, two adults $(\mathrm{A}+\mathrm{A})$, as well as with one adult and one infant $(\mathrm{A}+\mathrm{I})$. In turn, because of the low juvenile mortality rate, each of these three combinations should represent nearly $30 \%$ of the total of double burials. On the other hand, if we consider sex, an unbiased distribution would mean that in the double adult burials we should expect to find the same percentage of two women, two men, and a pair of individuals of different sex (33\% in each case).

However, these expected percentages are very far from the reality (Tables 1 and 2), to the point of discarding the "null hypothesis". ${ }^{22}$ Rather than a random selection, the double burials seem to favour the combination of man + woman, as against the alternatives involving two same-sex adults or two infants. Only the combination adult + infant comes near the expected values, with noticeable frequences at El Oficio, El Argar, and Peñalosa, but strong underrepresentation or even absence elsewhere (Gatas).

In short, through their frequency and the sex and age profile, double burials express a social selection. Roughly only one out of ten burials included a second interment and, perhaps most interestingly, the first occupant conditioned the profile of her/his later companion. In other words, one out of ten intramural burials was closely linked with a previous funerary rite. We suspect that discovering what made them differents would reveal a key aspect of Argaric society.

\subsection{Assessing sociological hypothesis: affinity or descent}

The relative high number of double burials with two adults leads us to think that they may have had some socially recognised affinity. In addi- 
tion, the fact that this relationship was ritually sanctioned gives an idea of its great social relevance, even more so if we consider how scarce the symbolic expressions left by Argaric society are.

The majority of the alliances included in the political category of "marriage" establish that men and women should relate with spouses of the opposite sex with whom they have no blood ties, i.e., in accordance with the principle of heterosexuality and the taboo against incest. If double burials symbolised heterosexual marital unions, which is the most accepted view in past scholarship, ${ }^{23}$ then possibly the funerary rite laid symbolic emphasis on the importance of founding new families, in this case monogamous and perhaps nuclear; that is, ideology would have had a special way of celebrating the establishment of ties between families and, in a way, the ability of each of them to show openness.

However, to validate this statement we need to test whether the men and women in double burials were contemporaneous. The observations that allow us to propose simultaneousness (articulated deposition of both skeletons with the members overlapping, i.e. when none of the dead bodies rests entirely above or below the other ${ }^{24}$ ) or temporal distance (displaced and disjointed -also known as "reduced"- deposition of one of the skeletons) are not usually published. Now even where the data are detailed it provides only arguments of relative chronology, which are insufficient to deal with the question. It is not enough to ascertain that a dead body was interred before or after the other: rather, it is essential to know how much time elapsed between the two. ${ }^{25}$ Therefore, there is a need for indicators of absolute chronology. In various publications of the 1990s, we pointed out that the radiocarbon dates of pairs of adults in double burials did not support the hypothesis of their contemporaneousness in life, but rather raised the possibility that they represented women and men separated by a minimum of two generations. ${ }^{26}$ If this is so, not even the coexistence in life should be taken for granted. From this observation we suggested that the relationship between the two individuals may have been one of kinship, e.g. between grandmother and grandson.

Our questioning of the marital relationship of these individuals opened a new source of interest. Further research has increased the data, with regard to the number of dates available and their geographical scale, which now includes several inland sites in Granada (Castellón Alto, Cuesta del Negro, Cerro de la Encina and Cerro de la Virgen). ${ }^{27}$ Cámara and Molina (2009) have dealt with the question of the differential chronology of the individuals in double burials in the light of these new radiocarbon series. After comparing semi-quantitatively the pairs of calibrated dates for each grave, they conclude that the generational difference hypothesis lacks support. They did not put forward the monogamous model, so that the social meaning of double burials would seem to be left up in the air.

However, if the mathematical comparison between the pairs of radiocarbon dates is approached in terms of probability, ${ }^{28}$ the discussion takes place on a solid ground. First, the number of double burials whose occupants probably died in a short time-span is not zero, as when we advanced the hypothesis in the 1990s. Nevertheless, the group of double burials whose occupants could hardly have lived contemporaneously to the degree expected in a situation of marital union still represents the greatest majority. Two-thirds of 23 pairs of radiocarbon dates show internal differences of at least three decades, and in most of these cases the difference is of no less than 60 years, which corresponds to at least two generations (as we suggested initially). It is worthwhile mentioning that the nitrogen and carbon isotopic analysis carried out so far, involving a large sample of Argaric skeletons, preclude a significant consumption of water resources ${ }^{29}$ and hence distorting radiocarbon results due to the "reservoir effect".

The idea that two adults buried together were contemporaneous was the basis for the first Argaric sociological hypothesis set out by Inchaurrandieta and the Sirets in the late nineteenth century. The proximity between the corpses, the combination of sexes and, at times, the care with which the bodies were deposited were linked to monogamy and, by extension, to nuclear families. However, as we have shown the temporal distance between most of the adults in double burials challenges their synchronicity. Thus, if we maintain the hypothesis that people buried in the same grave were kin, then their ties may have been genealogical (consanguineous affiliation).

While we are still waiting for DNA analyses capable of testing this proposal, ${ }^{30}$ the high number of double burials with a large temporal gap between the two interments gives the "genealogical hypothesis" an advantage over that of a political-marital relationship: upon death, politi- 
cal alliances might have been symbolic, but never effective, whereas blood ties find their place and meaning in generational succession. Consanguinity would justify those cases in which the time-span is wide and, accordingly, it would account for the few cases in which that distance seems to be shorter as extreme examples of the main tendency (e.g. the youngest grandmother buried alongside the oldest grandson, the two having died almost at the same time). Likewise, a consanguineous link would account best for the remaining double burials, i.e. those that contain an adult and an infant, or two infants, representing situations in which we can hardly be dealing with spouses.

It must be stressed that we know of no case in which an infant inaugurated a grave where later an adult was interred, ${ }^{31}$ which emphasise the respect for the principle of ancestry we suggest. Where we have knowledge of the sequence of inhumation and the biological profile of the occupants $^{32}$ (Table 3), we found two examples of simultaneous burial comprising an adult woman and an infant. ${ }^{33}$ In other cases, it might be premature to support a maternal link based on the adult + infant combination. Thus, of the seven burials with a child in which the gender of the adult occupant has been established, three contained men, a fourth burial a possible man, and the age of the infants included a newborn and a baby under the age of 3 months. ${ }^{34}$ Together with the uncertainty surrounding the time-span between the two inhumations, this makes it possible to extend the hypothetical parental ties to the combination grandfather/grandmother + granddaughter/grandson.

Additional observations and an independent reasoning might support the hypothesis of descent ties. At sites such as Gatas ${ }^{35}$ and La Bas$\operatorname{tida}^{36}$ :

1. there is usually no more than a double burial with a pair of adults per architectural unit and occupational phase (which, usually, spans more than a century), and

2. a double burial often determines the arrangement of other graves in close proximity in the same unit. ${ }^{37}$

In sum, it is difficult to imagine that double burials were the posthumous echo of institutionalised marital ties. Had it been so, we should expect this type of burials to be much more frequent. Rather, they may have been emblematic, namely symbols of a relationship of consanguinity and ancestry that lasted through several generations in certain housing units. In the meantime, other burials were gradually added following selection criteria that still remain unclear.

In any case, the key question has to do with absolute dating. In this regard it must be admitted that, although this has provided the strongest empirical support for our hypothesis, it is also in the radiocarbon dates that its greatest frailty lies. We refer not only to the degree of uncertainty of each date inasmuch as they consist of a distribution of probability, but mostly to the uncertainties connected with the radiocarbon analysis as an instrumental procedure, namely those factors which are poorly known or of difficult control and which might approximate or separate results regardless of the actual elapsed time between the first and the second inhumation. Does every bone in the human skeleton react equally in the process of isotopic fractionation? How can we detect and assess with greater precision the distortion caused by the consumption of variable portions of seafood affected by the reservoir effect? Do we have a precise idea of the scale of the experimental and measurement error in the radiocarbon analysis of bone collagen samples? An experimental programme based on multiple dating from samples of different bones from the same skeleton, applied to each of the two skeletons of a double burial might provide information for a more accurate assessment of the current debate. There is no doubt that, if the difference between the dates of the same individual are as wide as the one between them and the dates of the companion, then it might be best to postpone the social-parental debate until the technical procedures of analysis became more precise and reliable.

\subsection{Assessing the sociological hypotheses: matrilineality and matrilocality}

Other investigations, undertaken in parallel, suggest that women tended to stay in their place of birth throughout their lives, whereas men moved, probably when married, and therefore were not usually buried in the same place they were born. This hypothesis was based on a metric variability of skulls that was significantly higher in the male population of the site of El Argar vis-à-vis the female population ${ }^{38}$.

The hypothesis that the Argaric society may have followed matrilocal norms has not yet been tested by new osteological and isotopic research. 
Table 3. Argaric double burials with data on the sequence of deposition and biological profile of the occupants.

\begin{tabular}{|c|c|c|c|c|c|c|c|}
\hline $\begin{array}{l}\text { Burial } \\
\text { no. }\end{array}$ & Sex and age & Skeleton position & Order & \begin{tabular}{|l|} 
Burial \\
type
\end{tabular} & $\begin{array}{l}\text { Social } \\
\text { cat. }\end{array}$ & Goods & Bibliography \\
\hline $\begin{array}{l}\text { AR- } \\
824\end{array}$ & $\begin{array}{l}\text { M? 20/30 y } \\
\text { F } 12 / 15 \text { y }\end{array}$ & & F-M & $\mathrm{UR}^{\mathrm{D}}$ & 1 & $\begin{array}{l}\text { F5, F8, ESP, } \\
\text { EXT } \tilde{N}^{3 R}, \mathrm{BZT}, \\
\text { 5PD/AN, PD/ } \\
\text { AN*, COLL, } \\
\text { fauna }\end{array}$ & $\begin{array}{l}\text { Siret 1890: } 206 \text { y lám.69; Jacques } \\
\text { 1890: 480; Kunter } 1990\end{array}$ \\
\hline AYC-1 & $\begin{array}{l}M>20 y \\
F>20 y\end{array}$ & & F-M & CIMx & $3 *$ & $\begin{array}{l}2 \mathrm{~F} 5, \mathrm{PN}^{2 \mathrm{R}} \text {, } \\
\text { sickle chip and } \\
\text { tooth }\end{array}$ & Cuadrado 1945: 380 \\
\hline AY-44 & $\begin{array}{l}M>20 y \\
F>20 y\end{array}$ & $\begin{array}{l}\text { Supine with Lat L } \\
\text { extremities } \\
\text { Disarticulated and } \\
\text { over M }\end{array}$ & F-M & CIMx & 3 & $\begin{array}{l}\mathrm{F} 5, \mathrm{PN} \tilde{N}^{3 R}, \mathrm{PZ} \\
\text { fauna }\end{array}$ & $\begin{array}{l}\text { Unpublished (2014 Autonomous } \\
\text { University of Barcelona) }\end{array}$ \\
\hline BA-16 & $\begin{array}{l}\text { M 35/40 y } \\
\text { F 35/40 y }\end{array}$ & $\begin{array}{l}\text { Lat } \mathrm{L} \text {, over } \mathrm{F} \\
\text { Lat } \mathrm{R} \text {, under } \mathrm{M}\end{array}$ & F-M & UR & 3 & $\begin{array}{l}\text { F2, PÑ, PZ, } \\
\text { 24COLL }\end{array}$ & $\begin{array}{l}\text { Unpublished (2009 Autonomous } \\
\text { University of Barcelona) }\end{array}$ \\
\hline BA-40 & $\begin{array}{l}M>20 y \\
F>20 y\end{array}$ & $\begin{array}{l}\text { Lat } \mathrm{L} \\
\text { Disarticulated over } \\
\text { and under M }\end{array}$ & F-M & CIL & 1 & $\begin{array}{l}\text { ALB, PÑ, PZ, } \\
\text { fauna }\end{array}$ & $\begin{array}{l}\text { Unpublished (2009 Autonomous } \\
\text { University of Barcelona) }\end{array}$ \\
\hline BAJ-6 & $\begin{array}{l}\mathrm{M} \\
\mathrm{F} \\
\end{array}$ & Lat L & F-M & UR & 3 & F7, PÑ, PZ, 2PD & $\begin{array}{l}\text { Unpublished (field notes and } \\
\text { photographs of J. Evans 1950) }\end{array}$ \\
\hline $\begin{array}{l}\text { CA- } \\
018\end{array}$ & $\begin{array}{l}\text { M 20/25 y } \\
\text { F } 20 / 25 \text { y }\end{array}$ & $\begin{array}{l}\text { Lat } \mathrm{L} \\
\text { Disarticulated and } \\
\text { over M }\end{array}$ & F-M & $\mathrm{CO}$ & 4 & $\mathrm{~F} 4, \mathrm{AN}, \mathrm{COLL}$ & $\begin{array}{l}\text { Haro 2012: } 392 \text {; display panels } \\
\text { at the site }\end{array}$ \\
\hline $\mathrm{CV}-06$ & $\begin{array}{l}\text { M 25/30 y } \\
\text { F } 50-55 \text { y }\end{array}$ & & F-M & Chamber & 1 & $\overline{\mathrm{PN}}, \mathrm{AN}^{\mathrm{o}}$ & $\begin{array}{l}\text { Schüle 1967: 119; Cámara and } \\
\text { Molina 2009: 173; Botella and du } \\
\text { Souich, unpublished report }\end{array}$ \\
\hline EN-17 & $\begin{array}{l}\text { M 35/50 y } \\
\text { F 20/35 y }\end{array}$ & $\begin{array}{l}\text { Articulated } \\
\text { Disarticulated and } \\
\text { displaced }\end{array}$ & F-M & CIM & $3 *$ & $\begin{array}{l}\text { F1/F2, PÑ } \\
\text { alisador }\end{array}$ & $\begin{array}{l}\text { Romero 1988: } 145 \text { and tab. 1; } \\
\text { Lapuente 2008: tab. } 1 \text { and } 6\end{array}$ \\
\hline $\begin{array}{l}\text { FA- } \\
069\end{array}$ & $\begin{array}{l}\text { M 20/40 y } \\
\text { F 20/30 y }\end{array}$ & $\begin{array}{l}\text { Lat } \mathrm{L} \text {, partially } \\
\text { disarticulated } \\
\text { Lat } \mathrm{R}\end{array}$ & F-M & $\mathrm{CIL}$ & 3 & F5, PÑ, PZ & $\begin{array}{l}\text { Schubart 2012: 133-135 and láms. } \\
\text { 7, 50-c and } 36\end{array}$ \\
\hline $\begin{array}{l}\text { FA- } \\
075^{*}\end{array}$ & $\begin{array}{l}\text { M 55/65 y } \\
\text { F } 20 / 30 y\end{array}$ & $\begin{array}{l}\text { Lat L, but lower } \\
\text { extremities disarti- } \\
\text { culated } \\
\text { Disarticulated }\end{array}$ & F-M & $\mathrm{CO}$ & 1 & $\begin{array}{l}\text { F5, F6, ALB, } \\
\text { PZ, BZT }^{\circ}\end{array}$ & $\begin{array}{l}\text { Schubart et al. 1996: 57; Schubart } \\
\text { 2012: 139-140, láms. } 10 \text { and } 61\end{array}$ \\
\hline GT-33 & $\begin{array}{l}M>50 y \\
F>50 y\end{array}$ & $\begin{array}{l}\text { Lat } \mathrm{L} \\
\text { Disarticulated and } \\
\text { over M }\end{array}$ & F-M & CIL & 3 & F2, PÑ & $\begin{array}{l}\text { Castro et alii 1993: 18-19 and } \\
\text { fig. } 3\end{array}$ \\
\hline IL-1 & $\begin{array}{l}M>45 y \\
F 24-30 y\end{array}$ & $\begin{array}{l}\text { Lat } \mathrm{L} \\
\text { Disarticulated and } \\
\text { displaced }\end{array}$ & F-M & CIMx & $3 *$ & $\mathrm{PN}, \mathrm{BOT}^{\mathrm{MF}}$ & $\begin{array}{l}\text { López Padilla et alii 2006: 126- } \\
130 \text { and fig. } 54\end{array}$ \\
\hline $\begin{array}{l}\text { LMI- } \\
11\end{array}$ & $\begin{array}{l}\text { M 30/35 y } \\
\text { F 28/35 y }\end{array}$ & $\begin{array}{l}\text { Lat } \mathrm{L} \text {, under } \mathrm{F} \\
\text { Lat } \mathrm{R} \text {, disarticulated } \\
\text { and partially over } \mathrm{M}\end{array}$ & F-M & CIL & $3 *$ & PZ, BZT, fauna & $\begin{array}{l}\text { Martínez and Ponce 2002a: } 122 \\
\text { and fig. } 20\end{array}$ \\
\hline LTI-2* & $\begin{array}{l}\text { M 35/45 y } \\
\text { F 35/40 y }\end{array}$ & $\begin{array}{l}\text { Lat Iz } \\
\text { Partially } \\
\text { disarticulated and } \\
\text { over M } \\
\end{array}$ & F-M & FOC & $3 *$ & $\mathrm{PN}$ & $\begin{array}{l}\text { Martínez and Ponce 2002b: } 155 \\
\text { and lám. } 5\end{array}$ \\
\hline NE-31 & $\begin{array}{l}M>20 y \\
F>20 y\end{array}$ & & F-M & FOS & 1 & $\begin{array}{l}2 \mathrm{~F} 5, \mathrm{~F} 7, \tilde{P N}^{4 R} \\
\text { BZL, BZT* } \\
\text { AN/PD }{ }^{\circ}, 6 A N / \\
\text { PD* } 17 \text { COLL }\end{array}$ & $\begin{array}{l}\text { Cámara and Molina 2009: 180; } \\
\text { Cámara 2001: tab. } 28\end{array}$ \\
\hline
\end{tabular}




\begin{tabular}{|c|c|c|c|c|c|c|c|}
\hline CE-21 & $\begin{array}{l}\text { M } 22 / 24 \text { y } \\
\text { F } 16 / 17 \text { y }\end{array}$ & $\begin{array}{l}\text { Lat } \mathrm{L} \\
\text { Lat } \mathrm{R} \text {, partially } \\
\text { over M }\end{array}$ & $\mathrm{M}-\mathrm{F}$ & $\mathrm{FO} / \mathrm{CO}$ & 2 & $\begin{array}{l}\text { 2F1, 2F2, } \\
\text { 2F4, F7, 2PÑ, } \\
6 \mathrm{BZT}^{*}, 3 \mathrm{AN} / \\
\mathrm{PD}^{*}, \mathrm{AN}^{*}, \\
\text { 2PD*, 25COLL, } \\
\text { Fauna }\end{array}$ & $\begin{array}{l}\text { Aranda et alii } 2008: 237-245 \text {, figs. } \\
8-10 \text { and láms. VIII-XI }\end{array}$ \\
\hline AY-15 & $\begin{array}{l}\mathrm{M} \\
\mathrm{F}\end{array}$ & $\begin{array}{l}\text { Disarticulated } \\
\text { Semi-extended } \\
\text { supine with knees } \\
\text { bended up }\end{array}$ & $\mathrm{M}-\mathrm{F}$ & CIL & 5 & & $\begin{array}{l}\text { Unpublished ( } 2013 \text { Autonomous } \\
\text { University of Barcelona) }\end{array}$ \\
\hline AY-24 & $\begin{array}{l}\mathrm{M} \\
\mathrm{F}\end{array}$ & $\begin{array}{l}\text { Disarticulated and } \\
\text { partially over } \mathrm{F} \\
\text { Lat } \mathrm{R}\end{array}$ & $\mathrm{M}-\mathrm{F}$ & CIL & 3 & $\begin{array}{l}2 \mathrm{~F} 5, \mathrm{PN}, \mathrm{PZ} \\
\text { fauna }\end{array}$ & $\begin{array}{l}\text { Unpublished (2013 Autonomous } \\
\text { University of Barcelona) }\end{array}$ \\
\hline AY-26 & $\begin{array}{l}\text { M 35/39y } \\
\text { F }>50 y\end{array}$ & $\begin{array}{l}\text { Disarticulated and } \\
\text { partially over F } \\
\text { Supine with Lat R } \\
\text { extremities }\end{array}$ & $\mathrm{M}-\mathrm{F}$ & CIMx & $3 *$ & $\mathrm{~F} 8, \mathrm{PN}^{2 \mathrm{R}}$, fauna & $\begin{array}{l}\text { Unpublished (2014 Autonomous } \\
\text { University of Barcelona) }\end{array}$ \\
\hline AY-27 & $\begin{array}{l}M>50 y \\
F>50 y\end{array}$ & $\begin{array}{l}\text { Disarticulated and } \\
\text { partially over F } \\
\text { Supine with Lat R } \\
\text { extremities } \\
\end{array}$ & $\mathrm{M}-\mathrm{F}$ & $\mathrm{UR}^{\mathrm{D}}$ & 4 & ${ }_{\mathrm{EXT}} \mathrm{F} 5$, fauna & $\begin{array}{l}\text { Unpublished (2014 Autonomous } \\
\text { University of Barcelona) }\end{array}$ \\
\hline AY-42 & $\begin{array}{l}\text { M 35/44 y } \\
\text { F 30/34 y }\end{array}$ & $\begin{array}{l}\text { Disarticulated and } \\
\text { laid aside } \\
\text { Lat R }\end{array}$ & M-F & CIL & 5 & Fauna & $\begin{array}{l}\text { Unpublished ( } 2014 \text { Autonomous } \\
\text { University of Barcelona) }\end{array}$ \\
\hline BA-12 & $\begin{array}{l}M>20 y \\
F 40-45 y\end{array}$ & $\begin{array}{l}\text { Lat } \mathrm{L} \text {, partially } \\
\text { under F } \\
\text { Lat } \mathrm{R} \text {, partially } \\
\text { over M }\end{array}$ & $\mathrm{M}-\mathrm{F}$ & Chamber & 3 & PÑ, PZ, fauna & $\begin{array}{l}\text { Unpublished (2009 Autonomous } \\
\text { University of Barcelona) }\end{array}$ \\
\hline BA-61 & $\begin{array}{l}\text { M 35-40 y } \\
\text { F 38-44 y }\end{array}$ & $\begin{array}{l}\text { Disarticulated and } \\
\text { over F } \\
\text { Supine with Lat R } \\
\text { extremities, under } \\
\text { M }\end{array}$ & $\mathrm{M}-\mathrm{F}$ & UR & $3 *$ & $\begin{array}{l}\text { F2, F5, PZ, } \\
\text { 3COLL, fauna }\end{array}$ & $\begin{array}{l}\text { Unpublished (2011 Autonomous } \\
\text { University of Barcelona) }\end{array}$ \\
\hline CV-30 & $\begin{array}{l}\text { M } 21 \text { y } \\
\text { F 22-24 y }\end{array}$ & & $\mathrm{M}-\mathrm{F}$ & ¿? & 5 & & $\begin{array}{l}\text { Cámara and Molina 2009: 173; } \\
\text { Delgado } 2013\end{array}$ \\
\hline $\begin{array}{l}\text { FA- } \\
058\end{array}$ & $\begin{array}{l}\text { M 40/60 y } \\
\text { F 20/30y }\end{array}$ & $\begin{array}{l}\text { Lat L, bottom of } \\
\text { burial and separated } \\
\text { from F by layer of } \\
\text { earth } \\
\text { Lat R, upper part of } \\
\text { the burial }\end{array}$ & M-F & $\mathrm{CO}$ & 1 & F5, ALB, PÑ & $\begin{array}{l}\text { Schubart 2012: 125, and láms. } 4 \\
\text { and } 63 \mathrm{~b} .\end{array}$ \\
\hline $\begin{array}{l}\text { FA- } \\
080\end{array}$ & $\begin{array}{l}\text { M 60/70 y } \\
\text { F 60/70 y }\end{array}$ & $\begin{array}{l}\text { Disarticulated and } \\
\text { displaced } \\
\text { Lat R }\end{array}$ & $\mathrm{M}-\mathrm{F}$ & $\mathrm{CO}$ & $3 *$ & $\mathrm{~F} 2, \mathrm{PN}$ & $\begin{array}{l}\text { Schubart 2012: } 142-143 \text { and láms. } \\
12,64-c \text { and } 38\end{array}$ \\
\hline GT-37 & $\begin{array}{l}M>47 y \\
F>50 y\end{array}$ & $\begin{array}{l}\text { Lat L } \\
\text { Lat R, partially } \\
\text { over M }\end{array}$ & $\mathrm{M}-\mathrm{F}$ & CIM & 5 & & Castro et alii 1994: $220-221$ \\
\hline LCT-5 & $\begin{array}{l}\text { M 20/30 y } \\
\text { F 35/39y }\end{array}$ & $\begin{array}{l}\text { Disarticulated and } \\
\text { partially over } \mathrm{F} \\
\text { Lat } \mathrm{R}\end{array}$ & M-F & UR & $3 *$ & $\mathrm{~F} 5, \mathrm{~F} 8, \mathrm{PN}^{3 \mathrm{R}}$ & Sánchez Pravía 2001: 20-21 \\
\hline NE-19 & $\begin{array}{l}\mathrm{M}>20 \mathrm{y} \\
\mathrm{F} 12 / 20 \mathrm{y} \\
\end{array}$ & & $\mathrm{M}-\mathrm{F}$ & FOS & $3 *$ & $\mathrm{~F} 5, \mathrm{PN}^{3 \mathrm{R}}$ & $\begin{array}{l}\text { Cámara and Molina 2009: 180; } \\
\text { Cámara } 2001 \text { tab. } 28\end{array}$ \\
\hline PD-1 & $\begin{array}{l}M>20 y \\
F 25-30 y\end{array}$ & $\begin{array}{l}\text { Disarticulated and } \\
\text { over F } \\
\text { Articulated under M }\end{array}$ & M-F & FO? & -- & $\begin{array}{l}\text { Undeterminable } \\
\text { (disturbed } \\
\text { burial) }\end{array}$ & $\begin{array}{l}\text { López Padilla } 2009 \text { and pers. } \\
\text { comm. }\end{array}$ \\
\hline PÑ-18 & $\begin{array}{l}\text { M 40/60 y } \\
\text { F } 40 / 60 \text { y }\end{array}$ & $\begin{array}{l}\text { Disarticulated and } \\
\text { dispersed } \\
\text { Articulated } \\
\end{array}$ & M-F & CIM & $3 *$ & $\mathrm{~F} 1, \mathrm{PN}^{2 \mathrm{R}}$ & $\begin{array}{l}\text { Alarcón 2010: 1000; Alarcón } \\
\text { 2012: 46-47 }\end{array}$ \\
\hline $\begin{array}{l}\text { CA- } \\
007\end{array}$ & $\begin{array}{l}\mathrm{M} \\
\mathrm{F}\end{array}$ & $\begin{array}{l}\text { Lat } \mathrm{L} \\
\text { Lat } \mathrm{R} \text {, partially } \\
\text { over M }\end{array}$ & $\mathrm{M}-\mathrm{F}$ & $\mathrm{CO}$ & $\geq 4$ & $\begin{array}{l}\text { One vessel } \\
\text { (according } \\
\text { to graphic } \\
\text { information) }\end{array}$ & $\begin{array}{l}\text { Molina et alii 1986: 358; Cámara } \\
\text { and Molina 2009; Al-Oumaoui } \\
\text { 2009: fig. } 14\end{array}$ \\
\hline
\end{tabular}




\begin{tabular}{|c|c|c|c|c|c|c|c|}
\hline BA-18 & $\begin{array}{l}\text { M1 22/27 } \\
\text { M2 20-25 }\end{array}$ & $\begin{array}{l}\text { Lat R } \\
\text { Lat L, partially over } \\
\text { M2 }\end{array}$ & $\mathrm{M}-\mathrm{M}$ & UR & 3 & $\begin{array}{l}\mathrm{F} 2,{ }_{\mathrm{EXT}} \mathrm{F} 5, \mathrm{HAC}, \\
\mathrm{PN}^{3 \mathrm{R}}, \mathrm{PD}^{*}, \\
\text { fauna }\end{array}$ & Lull et alii 2012: 65 \\
\hline AY-21 & $\begin{array}{l}\text { F } \\
\text { Inf new-born }\end{array}$ & $\begin{array}{l}\text { Lat } \mathrm{R} \\
\text { Lat } \mathrm{L} \text {, under and } \\
\text { over } \mathrm{F}\end{array}$ & Simult. & CIL & 3 & PÑ, PZ, fauna & $\begin{array}{l}\text { Unpublished (2013 Autonomous } \\
\text { University of Barcelona) }\end{array}$ \\
\hline CE-06 & $\begin{array}{l}\text { F } 30 y \\
\text { Inf } 4 \text { y }\end{array}$ & $\begin{array}{l}\text { Lat } \mathrm{R} \\
\text { Lat L, between F } \\
\text { arms }\end{array}$ & Simult. & FOS & $3 *$ & $\begin{array}{l}\text { Three vessels, } \\
\text { PZ }\end{array}$ & $\begin{array}{l}\text { Sánchez Romero 2008: } 14 \text { and } \\
\text { fig. } 1\end{array}$ \\
\hline AY-38 & $\begin{array}{l}\text { M } 35-40 \text { y } \\
\text { F } 25-27 \text { y }\end{array}$ & $\begin{array}{l}\text { Lat L } \\
\text { Supine with Lat R } \\
\text { extremities }\end{array}$ & Simult? & UR & 1 & $\begin{array}{l}\text { F1 F4 F5* } \\
\text { DIA }{ }^{A P *} \mathrm{PN}^{4 R *} \\
\text { PZ }^{*} 3 \mathrm{BZT}^{2 *} \\
4 \mathrm{DIL}^{2 \mathbf{0} / 2 *} \\
2 \mathrm{AN}^{2 *} 10 \mathrm{PD}^{7 *} \\
19 \mathrm{COLL} \text { Fauna }\end{array}$ & Lull et alii 2015: 61-62 \\
\hline PÑ-21 & $\begin{array}{l}\text { Ind }>20 y \\
\text { Inf } 4 / 5 \mathrm{y}\end{array}$ & Lat $\mathrm{R}$ & Ad-Inf & ¿? & ¿? & ¿? & Sánchez and Alarcón 2012: 76-77 \\
\hline MA-3 & $\begin{array}{l}\text { M 35/40 y } \\
\text { Inf 2,5/3,5 y }\end{array}$ & $\begin{array}{l}\text { Lat } \mathrm{R} \\
\text { Lat R, partially } \\
\text { over M }\end{array}$ & M-Inf & FOS & 4 & $\mathrm{PD}^{*}$ & Unpublished (J. A. Zapata 2007) \\
\hline BA-6 & $\begin{array}{l}\text { M 25/35 y } \\
\text { Inf new-born }\end{array}$ & $\begin{array}{l}\text { Supine with Lat L } \\
\text { extremities } \\
\text { partially } \\
\text { disarticulated and } \\
\text { over M }\end{array}$ & M-Inf & UR & 4 & $\begin{array}{l}\text { F4, F7, 2PD*, } \\
\text { 11COLL, Fauna }\end{array}$ & $\begin{array}{l}\text { Unpublished (2009 Autonomous } \\
\text { University of Barcelona) }\end{array}$ \\
\hline LMC-7 & $\begin{array}{l}\text { F } 25 / 30 y \\
\text { Inf } 4 / 5 \text { y }\end{array}$ & $\begin{array}{l}\text { Lat L } \\
\text { Lat L, partially } \\
\text { over F }\end{array}$ & F-Inf & $\mathrm{UR}^{\mathrm{D}}$ & 4 & F8, PD*, fauna & $\begin{array}{l}\text { Pujante and Martínez 2010; } \\
\text { Rihuete et alii } 2011\end{array}$ \\
\hline $\begin{array}{l}\text { FA- } \\
089\end{array}$ & $\begin{array}{l}\text { M? } 60 / 70 \text { y } \\
\text { Inf } 0-3 \mathrm{~m}\end{array}$ & $\begin{array}{l}\text { Lat R } \\
\text { Disarticulated }\end{array}$ & M?-Inf & $\mathrm{CO}$ & 4 & $\begin{array}{l}\text { F7, F8, PN } \tilde{N}^{3 R} \\
\text { BZT }\end{array}$ & $\begin{array}{l}\text { Schubart 2012: 149, lám. } 14 \text { and } \\
63 \text {-e }\end{array}$ \\
\hline $\begin{array}{l}\text { CA- } \\
121\end{array}$ & $\begin{array}{l}\text { M } 27 / 29 \text { y } \\
\text { Inf } 4 \text { y }\end{array}$ & Lat Iz & M-Inf & $\mathrm{CO}$ & 2 & $\begin{array}{l}4 \text { beakers } \\
\text { (includes F7), } \\
\text { HAC, PÑ, BZT, } \\
\text { 3AN* } \\
\end{array}$ & Molina et alii 2003 \\
\hline BA-71 & $\begin{array}{l}\text { F } 18-20 \text { y } \\
\text { Juv } 13-14 \text { y }\end{array}$ & $\begin{array}{l}\text { Disarticulated } \\
\text { Lat L }\end{array}$ & $\begin{array}{l}\text { M(Juv)- } \\
\text { Juv }\end{array}$ & UR & $3 *$ & $\begin{array}{l}\text { EXT } \mathrm{F} 5, \mathrm{~F} 8 \\
\mathrm{EXX}^{3 \mathrm{P}}{ }^{3 R}, \mathrm{BZT}, \\
4 \mathrm{PD}^{1 *} 13 \mathrm{COLL}\end{array}$ & $\begin{array}{l}\text { Unpublished (2011 Autonomous } \\
\text { University of Barcelona) }\end{array}$ \\
\hline AY-30 & $\begin{array}{l}\text { Inf } 14-19 \mathrm{~m} \\
\text { Inf } 8-9 \mathrm{y}\end{array}$ & $\begin{array}{l}\text { Disarticulated and } \\
\text { partially under } \\
\text { another infant } \\
\text { Lat L }\end{array}$ & Inf-Inf & FOS & 5 & & $\begin{array}{l}\text { Unpublished (2014 Autonomous } \\
\text { University of Barcelona) }\end{array}$ \\
\hline BA-23 & $\begin{array}{l}\text { Inf 9-11 m } \\
\text { Inf 7-10 m }\end{array}$ & $\begin{array}{l}\text { Disarticulated and } \\
\text { under another infant } \\
\text { Lat L }\end{array}$ & Inf-Inf & UR & 5 & & $\begin{array}{l}\text { Unpublished (2009 Autonomous } \\
\text { University of Barcelona) }\end{array}$ \\
\hline BA-65 & $\begin{array}{l}\operatorname{Inf} 2-3 \text { y } \\
\operatorname{Inf} \leq 1 \text { y }\end{array}$ & $\begin{array}{l}\text { Partially under } \\
\text { another infant } \\
\text { Supine with Lat R } \\
\text { extremities }\end{array}$ & Inf-Inf & $\mathrm{UR}^{\mathrm{D}}$ & 4 & $\begin{array}{l}\mathrm{F} \text { F1, PD, } \\
\text { 16COLL }\end{array}$ & $\begin{array}{l}\text { Unpublished (2011 Autonomous } \\
\text { University of Barcelona) }\end{array}$ \\
\hline
\end{tabular}

\section{Caption:}

The burials with ${ }^{14} \mathrm{C}$ included in the Bayesian statistical analysis (Lull et alii 2013) are shown with grey shading.

Burial: $\mathrm{AR}=\mathrm{El}$ Argar; AY = La Almoloya, series excavated by the Autonomous University of Barcelona; AYC = La Almoloya, series excavated by Cuadrado; BA = La Bastida, series excavated by the Autonomous University of Barcelona; BAJ = La Bastida, series excavated by Jordá and Evans; $\mathrm{CA}=$ Castellón Alto; $\mathrm{CE}=$ Cerro de la Encina; $\mathrm{CV}=$ Cerro de la Virgen; EN = Cerro de la Encina; FA $=$ Fuente Álamo; GT = Gatas; IL = Illeta dels Banyets; $\mathrm{LCT}=$ Lorca Castle series; $\mathrm{LMC}=$ Lorca, Madres Mercedarias convent series LMC = Lorca, Madres Mercedarias Church series; LTI = Lorca, C/ Los Tintes series; MA = Monteagudo; NE = Cuesta del Negro; PD = Cabezo Pardo; PÑ = Peñalosa.

Sex and age: $\mathrm{M}=$ male; $\mathrm{F}=$ female; $\mathrm{y}=$ years; $\mathrm{m}=$ months; $\mathrm{Inf}=$ child; Juv = juvenile.

Position $:$ Lat $=$ lateral $\mathrm{L}=$ on the left side; $\mathrm{R}=$ on the right side.

Burial type: $\mathrm{CIL}=$ cist of stone slabs; $\mathrm{CIM}=$ masonry cist; $\mathrm{CIMx}=$ cist of mixed bonding; $\mathrm{CO}=$ small rock-cut tomb; $\mathrm{FOS}=$ simple pit; $\mathrm{UR}=$ burial jar; $\mathrm{UR}^{\mathrm{D}}=$ two burial jar with their mouths facing each other.

Social cat.: according to the proposal of Lull and Estévez (1986); * category 3 without the standard pair of tools. 
Goods: $\mathrm{ALB}=$ halberd; $\mathrm{AN}=$ ring; $\mathrm{BOT}=$ button; $\mathrm{BZL}=$ sharpener ('archer wristguard'); $\mathrm{BZT}=$ bracelet; $\mathrm{COLL}=$ necklace (preceded by the number of beads); DIA ${ }^{\mathrm{AP}}=$ diadem with discoid appendix; DIL = ear stretcher; ESP = sword; EXT (sub-index)= external grave goods; F1, F2, F3, F4, F5, F6, F7 and F8 (ceramic vessels according to the traditional typology); HAC = axe; MF (super-index) = ivory; $\mathrm{PD}=$ earring; $\mathrm{PD} / \mathrm{AN}=$ earring or ring; $\mathrm{PN}=$ dagger; $\mathrm{PZ}=$ awl; $\mathrm{R}$ (super-index) = rivet, preceded by the number of such pieces in the respective object; ${ }^{\circ}$ (super-index $)=$ gold; * $($ super-index $)=$ silver.

$\boldsymbol{F A - 7 5 *}$ : Although in the recent monograph dedicated to the necropolis of Fuente Álamo Schubart (2012: 139-140) corrects the interment sequence proposed previously (Schubart et alii 1996), we believe that the indications of displacement in the female skeleton are consistent with it being the first entombment, a circumstance which in turn is supported by the radiocarbon dates of the two skeletons.

LTI-2*: Publication has some misprints affecting both biological profile and position which are corrected here according to the original anthropological study (by Buikstra \& Rihuete).

The results obtained for Gatas are inconclusive, since even though the strontium isotopes values appear to indicate a local characterisation of all analysed individuals, the oxygen isotope values show great differences with regard to the faunal collection that defines the local background. ${ }^{39}$ Conversely, the preliminary results from La Bastida and La Almoloya ${ }^{40}$ indicate an isotopic differentiation that allows us to discard the possibility of population movements between the two settlements.

Along with the matrilocality hypothesis came another that postulates principles of matrilinearity, after we observed that, in most of the graves containing two adults, it was the woman who was interred first. ${ }^{41}$ This was interpreted as a ritual acknowledgement of the leading women role in the founding of social relationships. However, this proposal must be reconsidered in the light of new findings, as the number of burials in which the man was interred first has increased significantly to the point of equalling the number of opposite cases (Table 3). Although the unpublished data from several sites of the Granada inlands need yet to be considered, there are no chronological or geographic factors to account for the initial deposition of a woman or a man in double graves. In this light, we cannot discard the possibility of a bilateral affiliation.

On the other hand, if we are to obtain well preserved samples, the DNA analyses could afford relevant data on the degree of kinship affiliation between the individuals in double burials. Another venue to be explored is the analysis of craniodental epigenetic traits, ${ }^{42}$ although the viability of these studies is restricted to well-preserved and archaeologically well-contextualised skeletal collections.

Nevertheless, another arguments might support a primacy of the descent through the female line. First, the copper awl, the item reserved to many women of the middle and upper classes, is found in children tombs more often than the axe, which is its counterpart in the adult male grave goods. ${ }^{43}$ Thus, the early and larger social recognition of these possible young girls (based on their association with the awl), in regard to young boys of their same class, might indicate a leading role of females in the lines of descent.

Second, beyond the implications of a specific item, as is the case with awls, the composition of the offerings of the double burials allows new approaches in spite of the archaeological problems raised by these contexts. In this sense, it is not always possible to assign specific items to one or the other individual with certainty, due to the taphonomic displacements. Moreover, the reopening of a grave at the time of the second interment presented the opportunity to reposition or remove items that had been deposited with the first corpse. On the other hand, we must consider the possibility that the grave goods were not individually assigned, but rather meant for the two occupants.

Despite these limitations, we have explored the composition of the grave goods in double burials in search of arguments in relation to the issues that concern us here. The first approach to the sample of 99 burials with reliable osteological and archaeological data ${ }^{44}$ shows that most of them have some kind of grave goods ( $\mathrm{n}=89$ or $92 \%$, against only 8 or $8 \%$ that lack any goods). This might fit well with the expected results if we take into account that, as the number of individuals in regard to the typical single burial doubles,

\begin{tabular}{|c|c|c|c|c|c|}
\hline Category & $n$ & $\mathrm{n}$ & $\%$ & $\mathrm{n}$ & $\%$ \\
\hline 1 & 12 & & 151 & \multirow{4}{*}{75} & \multirow{4}{*}{$76 \%$} \\
\hline 2 & 3 & 15 & 15,1 & & \\
\hline 3 & 35 & \multirow{2}{*}{60} & & & \\
\hline *3 & 25 & & 60,6 & & \\
\hline 4 & 14 & \multirow{2}{*}{24} & \multirow{2}{*}{24,2} & \multirow{2}{*}{24} & \multirow{2}{*}{$24 \%$} \\
\hline 5 & **10 & & & & \\
\hline
\end{tabular}

Figure 2. Classification of the analysed sample according to the category of grave goods. $* 3=$ presence of PÑ (PZ or HAC may be absent). ** 2 burials have a certain item that is not normative (necklace, stone axe). 


\begin{tabular}{|l|r|rr|lr|rr|}
\hline Category & N & \multicolumn{2}{|l|}{$\begin{array}{l}\text { Male + female } \\
\text { items }\end{array}$} & $\begin{array}{l}\text { Exclusively } \\
\text { male items } \\
\text { (ALB, ESP, } \\
\text { HAC) }\end{array}$ & $\begin{array}{l}\text { Exclusively } \\
\text { female items } \\
\text { (DIA, PZ) }\end{array}$ \\
\hline 1 & 10 & 3 & $\begin{array}{r}2 \text { ALB+PZ; } \\
1 \text { ESP DIA } \\
\text { PZ }\end{array}$ & 6 & 4 ALB; & 1 & $\begin{array}{r}1 \text { DIA } \\
\text { PZ }\end{array}$ \\
\hline 2 & 2 & 1 & $1 \mathrm{HAC}$ PZ & 0 & & 1 & $1 \mathrm{PZ}$ \\
\hline 3 & 35 & 0 & & 8 & $8 \mathrm{HAC}$ & 27 & $27 \mathrm{PZ}$ \\
\hline TOTAL & 47 & 4 & $8 \%$ & 14 & $30 \%$ & 29 & $62 \%$ \\
\hline
\end{tabular}

Figure 3. Classification of double burials with osteological data and presence of an item associated exclusively to either sex according to the category of grave goods.

the probability that a given grave includes some offering increases proportionally.

This accumulative effect is also observed after classifying the grave goods assemblages following the categories of Lull and Estévez (1986) (Fig. 2). Now, the percentage of wealthy burials is higher than those observed in previous works on single tombs (Lull et alii 2005). Thus, the grave goods that define the exploited classes (categories 4 and 5) have lowered roughly from $35-40 \%$ to $24 \%$, whereas those of the privileged classes were around two-thirds of the total and now reach three-fourths. Even so, the differences are not sharp enough to draw a much different picture of the Argaric society. This leads to our first conclusion, namely that the genealogical representation expressed in the double burials was part of a normative shared by all social classes. Possibly, it was rather a principle that involved all the population with a given degree of residential stability, regardless of their socio-economic condition.

At this point, we are in a position to expand the argument sketched above regarding the awl and its implications for the matrilineal hypothesis. Taking into account the burials with some item associated exclusively with either sex $(n=$ 47; awl and diadem for women: halberd, sword and axe for men) (Fig. 3), we observe that $62 \%$ of them included only an object exclusive to females, more than the double of those containing only some item restricted to males (30\%). An additional $8 \%$ contained both types of objects. When the three indicators are combined, the presence of items linked exclusively to women corresponds to roughly two-thirds. If, in addition, we bear in mind that the awl is possibly underrepresented due to the risk of going unnoticed during excavations because of its small size, the percentage might have been higher.

Hence, it seems reasonable to assume that women had a leading role in the symbolising of the genealogical relations expressed in the adult + adult double burials. In the analysis described so far, the concepts of genealogical relationship, place of residence and female sex tend to converge with more strength than any other notion related to males. In this respect, the initial inhumation of a man in half of the double burials might correspond to the ethnographic figure of the maternal uncle in a matrilineal order.

To sum up, the Argaric funerary practices seem to have undervalued the symbolic expression of marital heterosexual ties, instead favouring relationships of intergenerational descent in which the sexual variable was relevant. These would have been the relations that governed Argaric decisions regarding the distribution of the social subjects and, perhaps, the shaping of ideologies. The basic reference would have been a blood genealogy, probably transmitted and named through female lines, and settled within private households or, at most, a local sphere (settlement). No imagery would have been required to reinforce or remember a deeply conservative reality. It would have been enough to remember who was lying under the floor and ensure that its couple was not of the same sex, perhaps as a concession of the prevailing genealogical principle to the reproductive sphere.

Regardless of the future of this research, we must draw a lesson: knowledge advances through the formulation of risky hypotheses that raise new and sometimes complex questions. If we neglect this principle, we may fall prey to the complacency of platitudes, while failing to see that they 
carry no lesser uncertainties than those faced by a controversial hypothesis.

\section{The archaeological visibility of women and men}

The "sexing of the past" arose from the need to lend a voice to women as constructors of history in at least the same measure as men. It was put forward as a feminist proposal ${ }^{45}$ that pressed everyone to consider that the silencing on the role of women in history was, and still is, a strategy of the patriarchy, or at least of men, to rule the world. The sexing of the past from an archaeological perspective aimed the identification of women in the production, distribution and consumption of objects and subjects. It may appear that the difficulty was to identify women, but actually identifying women is just as difficult as identifying men. The generic primacy of men is more of a discursive theft than the effect of a verified reality.

To assess the tasks carried out traditionally by women according to history and ethnography should not imply accepting the universal predominance of women in those tasks. However, this last assumption is usually the starting point of texts that speak of "maintenance activities", a catch-all term that encompasses a wide range of tasks from making a dress, to comforting a child, to cooking. ${ }^{46}$ To assume that women carried out a whole series of "maintenance activities", on a "daily" basis or not, ${ }^{47}$ means viewing the past through a set of prejudices of the same kind that led patriarchal archaeologies to fill the past with man hunters, smiths, warriors, sailors, shamans, priests, merchants and rulers. Under the umbrella of "gender archaeology", which is not synonymous with feminist archaeology, "maintenance activities" have helped to break the invisibility of women in the archaeological discourse, but at the cost of keeping them with children raising, inside the house, and in the kitchen, i.e. the same care-giving and domestic spheres to which the patriarchal order has relegated them. If the only difference between one or the other perspective is the explicit awareness of the same prejudice and its positive appreciation against the oppressive silence of the Father, then we should simply consider both as the object of a possible sociology of academic thought, instead of a sociology of the human relationships in the past; little will have been accomplished if trying to make women visible in the past as the protagonists of certain maintenance activities is going to result in a corroboration of patriarchal prejudices.

Archaeology deals with the things that societies produce. Now these things lack sex/gender, even when they act as subjects that define us. ${ }^{48}$ We can establish that certain groups of things are linked to certain groups of individuals, but even this statement faces problems. Let us review them.

We may think, for instance, that certain objects were made by women, distributed by men and, ultimately, consumed by men and women. There can be plenty of such combinations, especially if we recognise that the social construct of sex (gender) is contingent and does not necessarily imply a neat binary distinction. Thus, how can we associate objects with any of the sexes? How are we to establish a clear distinction of production/sex, distribution/sex and consumption/sex if both men and women take part in this chain? Objects are basically social and the social sphere includes both sexes, even if one of them wishes to impose its primacy and exploits the other. The production of objects is carried out by men and women, and their products pass through both depending on the social division of labour and the rules of property. In general, it is hardly difficult to detect something unambiguously feminine or masculine (except pregnancy and breastfeeding), although we can approach who made a given thing, who contributed to its distribution, and who used or consumed it. Objects have a history and make history; they create and act as subjects of social life. ${ }^{49}$ Even if we would think that objects as material entities cannot be sexed, but rather the thoughts and ideologies they provide to those who make and/ or consume, we should bear in mind that these ideas can be known and shared even by other social groups.

Human remains show physical traits that allow identifying the sex in a high number of cases. ${ }^{50}$ Bodies can also be seen as social objects that have been used, and which have suffered or enjoyed the living contexts that society granted them. We are now on a much less thorny research: the asymmetries in pathologies, diet, physical wear and life expectancy are ways of learning about these other social-situational relations that society imprinted on its protagonists.

Objects make us different depending on who produces, distributes and uses them. This formative ability lie more in the use and/or possession 
of the objects than in the activities tied to their production and distribution. Thus, we can engage in the task of seeking a connection between social objects and sexed bodies from the perspective of use or consumption. As the most definitive kind of object deposition is the one that takes place upon death, the items that accompany the body of a man or a woman, as chosen by whoever performed the funerary practices, might express a particular relationship with the social activity of the dead when it was alive.

The differences in the grave goods of men, women and infants in the Argaric society have allowed different readings in terms of gender. The most common and widespread of them is based on the association -supported by bioarchaeological research- between weaponry (halberds, short swords or long daggers, axes ${ }^{51}$ and long swords) and men, from which women are excluded. ${ }^{52}$ Elsewhere we put forward the hypothesis that some male grave goods varied throughout time..$^{53}$ Thus, short swords and long daggers were replaced by long swords, which widened the reach of the cut, hack and jab. At the same time, by combining a blunter blow and the ability to hack, axes improved the efficiency of halberds. On the other hand, the awl, either in the company of a dagger/ knife or without it, was ascribed to women of the intermediate and upper classes throughout the Argaric period. ${ }^{54}$ The detail that put into question this continuity in the grave goods of women of the dominant class is the inclusion of silver, gold or electrum diadems, always in small numbers, in the late Argaric period. ${ }^{55}$ Given the uncertainty surrounding the sexing of children through their skeletons, the presence of axes and awls in children burials might indicate that in such cases we have boys and girls, respectively.

We cannot claim that the objects deposited in the burials were produced by their occupants during their lifetime, nor taking for granted a situation of prior daily use, but it is possible to assume that there is some sort of meaningful connection. What does it signify? It will be difficult to apply deductive rules: we cannot draw the conclusion that the awl, for instance, signifies "female identity" if this tool is not attested in all women burials, nor assume that they were in charge of their production because some women have it in their graves, nor even state that all women used it during their lives. What might seem like an unmistakable attribute of gender ${ }^{56}$, might more likely be an indicator of class (otherwise, it should be concluded that women interred without an awl did not deserve to be called "women").

But what might be the social implication of demonstrating through independent evidence the exclusive connection between the use of awls and -at least- some women? The answer is to assign a social value to labour. The latter goes beyond the individual and is part of a social production. There are no female -or male- specific tasks but motherhood. Discover which groups take on which tasks, and when, would be a first step to approach an investigation, no doubt difficult, about the role and visibility of both sexes. It has been proposed that the occurrence of tools such as awls and knives in female burials of the middle and upper classes might represent their role in the execution and/or control of the final stages of textile production, which is considered a crucial element in the process of materialization of surplus value in the Argaric economic system. ${ }^{57}$

\subsection{The treatment of the male and female body}

According to the funerary record, it seems clear that gender distinction was relevant in the Argaric society. Besides the ascription of certain grave goods (which denote class differences first of all), the very arrangement of the corpse inside the grave seems to have been defined by the sex/gender of the deceased. ${ }^{58}$ Table 4 summarizes the data on the position of 130 osteologically sexed skeletons from the whole Argaric territory ${ }^{59}(n$ women $=68 ; n$ men $=62)$. The bodies were in a flexed position with the lower extremities lateralised, regardless of whether they lay on the back or one of the $\operatorname{sides}^{60}$ (Fig. 4). A survey reveals that most women were deposited on their right side $(85 \%$; 58 out of 68 cases), whereas most men appear on their left side ( $74 \%$; 46 out of 62 cases). These trends are statistically significant. ${ }^{61}$

The cases that do not conform to the main trends (men on the right side and women on the left) reveal no significant correlations with other variables. Social class, for instance, does not seem to be a key criterion, since we find indistinct examples in both the male and female groups with grave goods of the first category. ${ }^{62}$ We also fail to see chronological determinants, ${ }^{63}$ and both the type of funerary container and the number of occupants (single or double burial) appear to be random factors. Therefore, there are rea- 

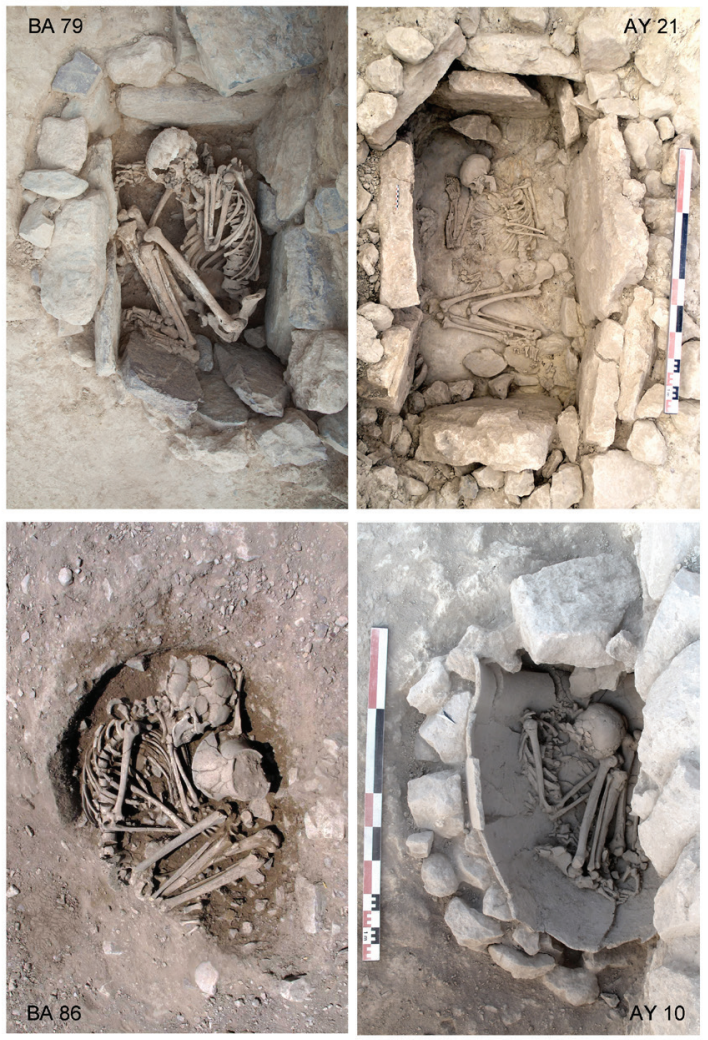

Figure 4. Main trends of body position according to sex. Up: female burials; below: male burials. $\mathrm{BA}=$ La Bastida; AY = La Almoloya.

sons to conclude that the positioning of the body depended above all on the sex/gender of the deceased.

Sexual differentiation in body position was not an idiosyncrasy of Argaric society. This kind of normative practice is attested among the Corded Ware groups in central-eastern and northern Europe around 2900-2800 cal BCE ${ }^{64}$ men being usually buried on their right side and women on the left. The expansion of the Bell Beaker horizon meant the reversing of this norm around $2600 / 2500$ cal BCE, so that women were now positioned on the right side and men on the left. This differentiation persisted throughout the second half of the third and the beginning of the second millennia cal $\mathrm{BCE}$, from the Atlantic to the Upper and Middle Danube, with the exception of Slovakia, where some groups maintained the old Corded Ware norm. ${ }^{65}$

We have undertaken a review, no doubt incomplete and only for exploratory purposes, of a sample of single and double burials attested in

\begin{tabular}{|l|l|l|}
\hline Male & Right & Left \\
\hline & $\begin{array}{l}\text { Fuente Olmedo (VA) } \\
\text { La Vital, assemblage 11 (V) } \\
\text { Rincón de Soto (LO) } \\
\text { Valle de las Higueras 5 (TO) }\end{array}$ & $\begin{array}{l}\text { Arenero de Soto (M) } \\
\text { Cerro de la Cabeza 4 (AV) } \\
\text { Cerro de las Viñas (MU) } \\
\text { El Hundido I y III (BU) } \\
\text { Humanejos UE1938 y UE1853 (M) } \\
\text { Juan Fco. Sánchez (M) } \\
\text { La Vaquera (SG) } \\
\text { Las Matillas UE100 (M) } \\
\text { Molinos de Papel 2 (MU) } \\
\text { Tres Montes (NA) }\end{array}$ \\
\hline Female & $\begin{array}{l}\text { C. Yeseras I1/F-492 (M) } \\
\text { C. Yeseras, small rock-cut tomb 1, } \\
\text { hut 5 (M) } \\
\text { La Vital, assemblage 10 (V) } \\
\text { Humanejos UE1853 (M) } \\
\text { Santioste (ZA) }\end{array}$ & \\
\hline
\end{tabular}

Figura 5. Position of individuals in single burials dated to between ca. 2500-2000 cal BCE in different regions of the Iberian Peninsula (according to Alonso 2013, Andrés et alii 2002, Ayala 1991, Blasco et alii 2005, Bueno et alii 2005, Fabián and Blanco 2012, García Puchol et alii 2011, Garrido et alii 2005, Gómez Pérez et alii 2011, Liesau et alii 2015, Pujante 2005, Ríos 2011, Valera 2014).

different regions of Iberia and dated to the second half of the third millennium BCE (Fig. 5). Despite the small size of the sample, it is interesting to note that most men were lying on the left side, whereas women rested on the right. This is close to the Bell Beaker norm and might be a precedent followed by the Argaric communities.

A systematic approach to the deposition of dead bodies of both sexes in the Iberian groups contemporaneous with the Argaric society remains desirable. The funerary customs seem to have been very heterogeneous, as much at the regional level as between individual cemeteries. ${ }^{66}$ Thus, there is no hint of a peninsular koine as regards this aspect, although in some cases, such as Las Cruces (Seville), ${ }^{67}$ the regularity and similarity to the Argaric pattern is striking. Thus, we cannot discard the possibility of interesting patterns when more detailed data will be available.

Now, although the funerary treatment according to sex proves to be patterned in the Argaric territory, we should keep in mind that $13 \%$ of the women and $27 \%$ of the men, i.e. nearly one-fifth of the adult population, were not buried according to the prevailing norms. It is therefore recommendable not to cast aside this unconforming group but, rather, to go more deeply into its archaeological contexts and attempt to account for its specificity. Hence, at Fuente Alamo, where the high percentage of men resting on their right side (Table 4) is striking, Schubart ${ }^{68}$ has suggested that only warriors and men old enough to behave as such would have been placed on the left side, whereas men too old (burials 89 and $95^{69}$ ), too 
Table 4. Argaric burials with data on body position and sex.

\begin{tabular}{|c|c|c|c|c|c|c|c|c|c|}
\hline Site & Province & & $\mathbf{M}$ & & & & & Burials & Bibliography \\
\hline & & $\mathbf{N}$ & $\mathbf{L}$ & $\mathbf{R}$ & $\mathbf{N}$ & $\mathbf{L}$ & $\mathbf{R}$ & & \\
\hline Illeta dels Banyets & Alicante & 2 & 2 & - & - & - & - & 1 and 3 & López Padilla et alii 2006. \\
\hline El Picacho & Almería & 1 & - & 1 & - & - & - & Pit 1 & $\begin{array}{l}\text { Hernández and Dug 1976; } \\
\text { sex estimation according to } \\
\text { our analysis (the bones are } \\
\text { kept in the Durán Vall-Llosera } \\
\text { Collection). }\end{array}$ \\
\hline Fuente Álamo & Almería & 12 & 8 & 4 & 11 & 1 & 10 & $\begin{array}{l}9,52,54,58,62,68,69, \\
70,75,80,81,85,89, \\
90,93,95,109,110,111 \\
\text { and } 112\end{array}$ & Schubart 2012: Abb. 11 * \\
\hline Gatas & Almería & 3 & 3 & - & 4 & - & 4 & $23 \mathrm{~B}, 33,37,41,42,44$ & $\begin{array}{l}\text { Castro et alii 1994: 205-280; } \\
\text { Castro et alii 1999: 9, láms. } \\
\text { V-VI; Castro et alii 2004: } 12 \\
\text { and fig. } 4 \text {. }\end{array}$ \\
\hline Herrerías-M.Iberia & Almería & 1 & 1 & - & - & - & - & No no. & $\begin{array}{l}\text { Pericot 1934: } 214-215 \text {; sex } \\
\text { estimation according to our } \\
\text { analysis (the bones are housed } \\
\text { in the Museo Arqueológico } \\
\text { Nacional, Madrid). }\end{array}$ \\
\hline Castellón Alto & Granada & 5 & 5 & - & 2 & - & 2 & $7,18,19,21,101$ and 121 & $\begin{array}{l}\text { Contreras et alii 1997: } 128 \\
\text { and 131; Molina et alii 2003; } \\
\text { Al-Oumaoui 2009: figs. 14-- } \\
\text { 16; Molina and Cámara 2009: } \\
\text { 212; Cámara and Molina 2009: } \\
\text { fig. } 3 \text { and lám. III; Cámara } \\
\text { and Molina 2010: 32; display } \\
\text { panels at the site. }\end{array}$ \\
\hline Cerro de la Encina & Granada & 4 & 2 & 2 & 5 & - & 5 & $\begin{array}{l}6,9,13,14,15,16,18 \\
\text { and } 21\end{array}$ & $\begin{array}{l}\text { Aranda and Molina 2006: tab. } \\
\text { 1; Sánchez Romero 2008; } \\
\text { Aranda et alii 2008; Al- } \\
\text { Oumaoui 2009: fig. } 31 \text { [turned } \\
\text { over], } 32 \text { and 34. }\end{array}$ \\
\hline Cerro de la Virgen & Granada & 1 & - & 1 & 1 & - & 1 & 21 & $\begin{array}{l}\text { Cámara et alii 2009: } 178 \text { and } \\
\text { lám. I. }\end{array}$ \\
\hline Cerro San Cristóbal & Granada & 3 & 2 & 1 & 3 & 1 & 2 & $8.1,8.2$ and 8.3 & Aranda et alii 2012 \\
\hline Bajil & Murcia & 1 & - & 1 & - & - & - & 6 & $\begin{array}{l}\text { Eiroa 1993-1994: fig. } 7 \text { and } \\
\text { lám. IX. }\end{array}$ \\
\hline Cabeza Gorda & Murcia & 1 & 1 & - & - & - & - & No no. & $\begin{array}{l}\text { Ayala and Tudela 1993: fig. } \\
2 \text { and lám. } 3 \text {; sex estimation } \\
\text { based on the photograph of } \\
\text { the skull in García Martínez } \\
\text { 1999: } 180 .\end{array}$ \\
\hline Cerro de las Viñas & Murcia & - & - & - & 1 & - & 1 & No no. & $\begin{array}{l}\text { Ayala et alii } 1999: 20 \text { and } 22 \text {; } \\
\text { Malgosa et alii 2004: fig 1. }\end{array}$ \\
\hline La Almoloya & Murcia & 7 & 5 & 2 & 17 & 2 & 15 & $\begin{array}{l}2,3,5,10,11,12,16,17, \\
18,21,24,26,27,29,31, \\
34,36,38,39,42,43,44 \\
\text { and } 45\end{array}$ & $\begin{array}{l}\text { Unpublished ( } 2013 \text { and } 2014 \\
\text { excavations of the Autonomous } \\
\text { University of Barcelona). }\end{array}$ \\
\hline La Bastida & Murcia & 12 & 10 & 2 & 12 & 1 & 11 & $\begin{array}{l}\text { SHP series 1944: } 15 \text {; } \\
\text { SHP series 1950: } 1 \text { and } 6 \text {; } \\
\text { Autonomous University } \\
\text { of Barcelona series: } 6,12 \text {, } \\
\text { 16, 18, 21, 33, 40, 48, 49, } \\
60,61,62,78,79,80,82 \text {, } \\
86 \text { and } 89\end{array}$ & $\begin{array}{l}\text { Del Val et alii 1947: } 96 \text { and } \\
\text { lám. XXII; diary of Posac } \\
\text { 1944; diaries of del Val } 1944 \\
\text { and 1945; unpublished works: } \\
\text { diary of Jordá 1950, diaries of } \\
\text { Evans } 1950 \text { and 2009-2013 } \\
\text { excavations of the Autonomous } \\
\text { University of Barcelona. }\end{array}$ \\
\hline
\end{tabular}




\begin{tabular}{|c|c|c|c|c|c|c|c|c|c|}
\hline Lorca & Murcia & 4 & 4 & - & 7 & 1 & 6 & $\begin{array}{l}\text { Convent Madres } \\
\text { Mercedarias series: } 5 \text { and } \\
\text { 7; Madres Mercedarias } \\
\text { Church series: } 3,5,11 \text { and } \\
\text { 14; C/ Los Tintes series: } \\
\text { 2; C/Zapatería series 11: } \\
\text { 8; Castle series: individual } \\
\text { cist without no. }\end{array}$ & $\begin{array}{l}\text { Martínez et alii 1995; Martínez } \\
\text { and Ponce 2002a and 2002b; } \\
\text { Pérez Richard 2007; Rihuete et } \\
\text { alii 2011. }\end{array}$ \\
\hline Los Cipreses & Murcia & 2 & 1 & 1 & 1 & 1 & - & 3,9 and 11 & Martínez et alii 1999. \\
\hline Monteagudo & Murcia & 3 & 1 & 2 & 4 & 2 & 2 & $1,2,3,4,5,7,8$ and 9 & $\begin{array}{l}\text { Unpublished (excavation by J. } \\
\text { A. Zapata 2007). }\end{array}$ \\
\hline All & & 62 & 45 & 17 & 68 & 9 & 59 & & \\
\hline & & & $73 \%$ & $27 \%$ & & $13 \%$ & $87 \%$ & & \\
\hline
\end{tabular}

\section{Caption:}

$\mathrm{L}=$ on the left side; $\mathrm{R}=$ on the right side. The position refers to the lateralisation of the lower extremities in regard to the trunk, regardless of whether the latter is also positioned on one of the sides (strict lateral deposition) or on the back (supine deposition).

* Lapse in the sexing of burial no. 80 (see Schubart 2012: 143).

young (burial 85 ) or physically disabled (burial 110) would have been deposited on the same side as women. Fuente Álamo, however, differs from other sites such as La Bastida (burials no. 18 and 78) and Monteagudo (burials no. 3 and 8 ), where four adult men without any trace of disabling injuries were buried on their right side. Furthermore, one of the interments of La Bastida provides other data of interest, which raise new questions and are presented below.

\section{Sex and transgression}

The combination man-woman in the double burials has few exceptions. One of them is burial no. 18 of La Bastida, excavated during the 2009 campaign in Room 3 (Fig. 6). ${ }^{70}$ It consists of a large ovoid jar containing the skeletons of two men buried in a short time-span according to archaeo-thanatological evidence (Fig. 7). ${ }^{71}$ The first was between 22 and 27 years old when he

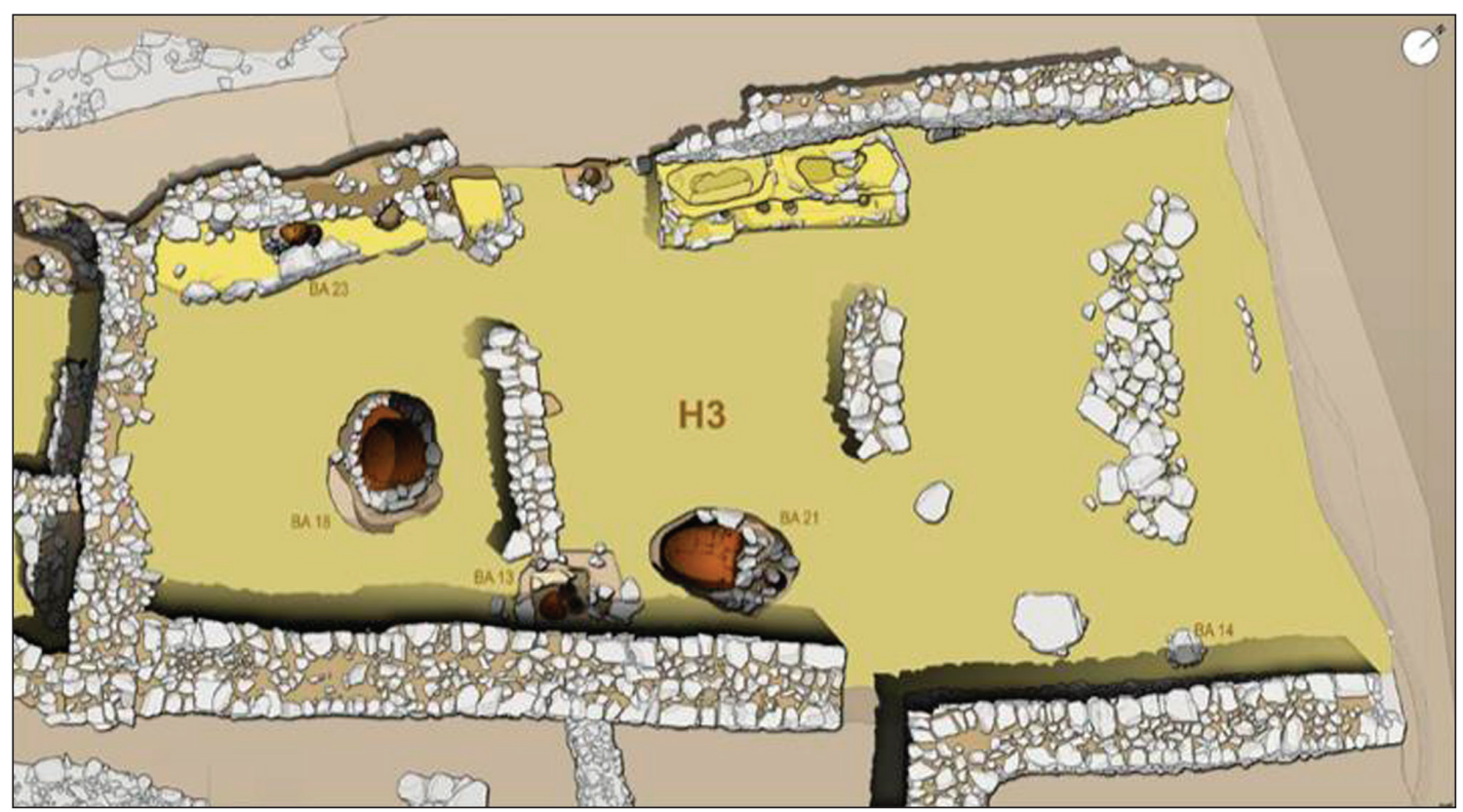

Figure 6. Plan of Room 3 of La Bastida, showing the location of burials no. 13, 18 and 21. 


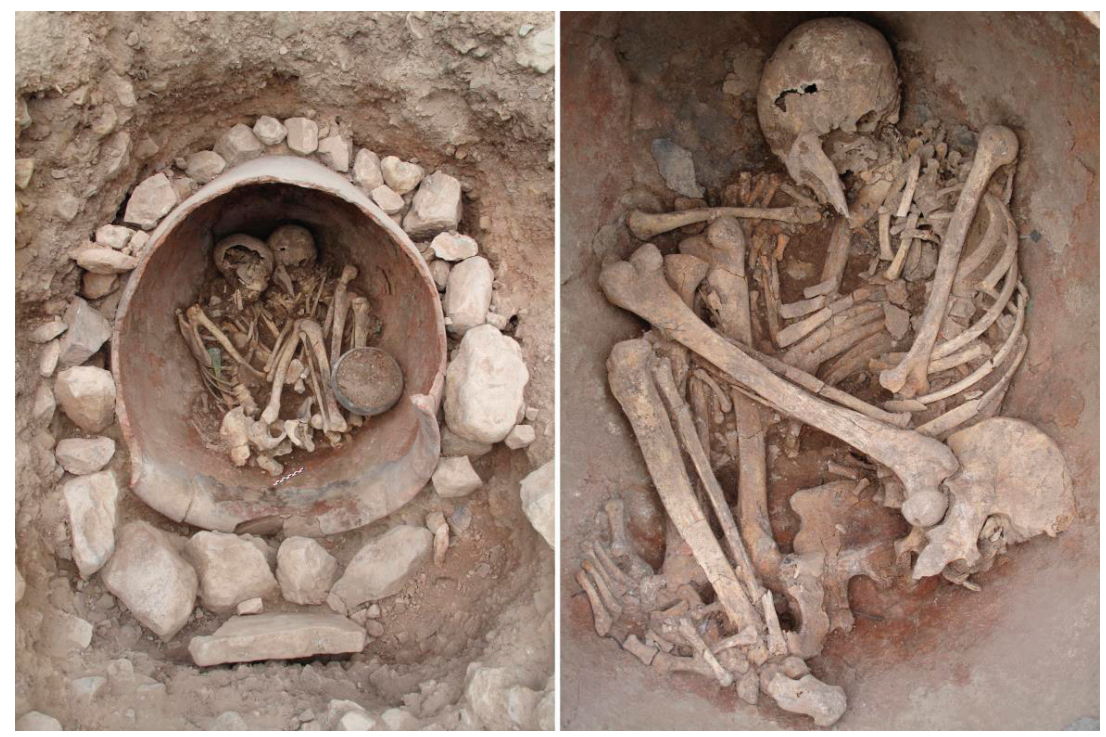

Figure 7. Burial no. 18 of La Bastida, with full views of the first (right) and second (left) interments.

died and was lying on his right side. The second man also died young, between 20 and 25 years of age, and was partially placed over the former on his left side and in the same face-to-face position seen in some burials with two adults of different sex. The first man had an axe behind his back and, the second, a dagger on his right side (probably hanging across his chest in a sheath). The pairing of these two objects makes up the typical grave goods of an Argaric man of the intermediate class. The second occupant wore a silver earring and over his legs were a calf's scapula ${ }^{72}$ and a bowl containing roasted barley. ${ }^{73}$ The first individual also had a meat offering: a sheep's leg. The carinated vessel placed as an external good could be associated with either body.

The two deeply-rooted practices of adult double burials seem to have been transgressed in burial no. 18: the generational difference between interments and the combination woman-man. Several possibilities can be entertained if this transgression was meant to emphasise a male affinity in life instead of a genealogical link. One is that these were two contemporary members of the same family, e.g. brothers. However, the fact that one of the men was positioned on his right side, as well as the likely temporal proximity between the two interments, invites us to consider other possibilities, such as the representation of a homosexual relationship. ${ }^{74}$ In this scenario, burial no. 18 would support the notion that El Argar was a sexually permissive society. However, be- fore consolidating this impression we should remember that La Bastida 18 is the only joint burial of two men attested in the core territory of El Argar; that we know only of three more cases in the peripheral north-western territories; and, above all, that we have no instance of a joint burial of two women.

There is other evidence from Room 3 that points to the singularity of this burial and, by extension, of its social context. At a short distance from La Bastida 18 another structure was found: a burial $\mathrm{jar}^{75}$ with no dead body inside. Judging by the size and type of the jar (Fig. 8), this cenotaph (burial no. 13) might be the simulation of an infant burial. ${ }^{76}$ The external grave goods included a carinated vessel, a cup and a small copper axe, i.e. the same type of exclusively-male object found in burial no. 18. It is hardly conceivable that the skeleton of this probable, or make-believe, boy was not preserved due to postdepositional disturbances, since the bones of a sheep's leg were found inside the jar.

A third burial completes the funerary evidence in this sector of Room 3. Like the previous burials, La Bastida 21 corresponds to a jar with a carinated vessel as an external offering, (Fig. 9). The grave goods found inside include a dagger and an awl, a necklace with a bone pendant and two stone beads, a cup and the foot of another placed over the right hip, and the leg of a sheep besides the back. The individual buried was a woman deceased at the age of 29-34 years. The body had 


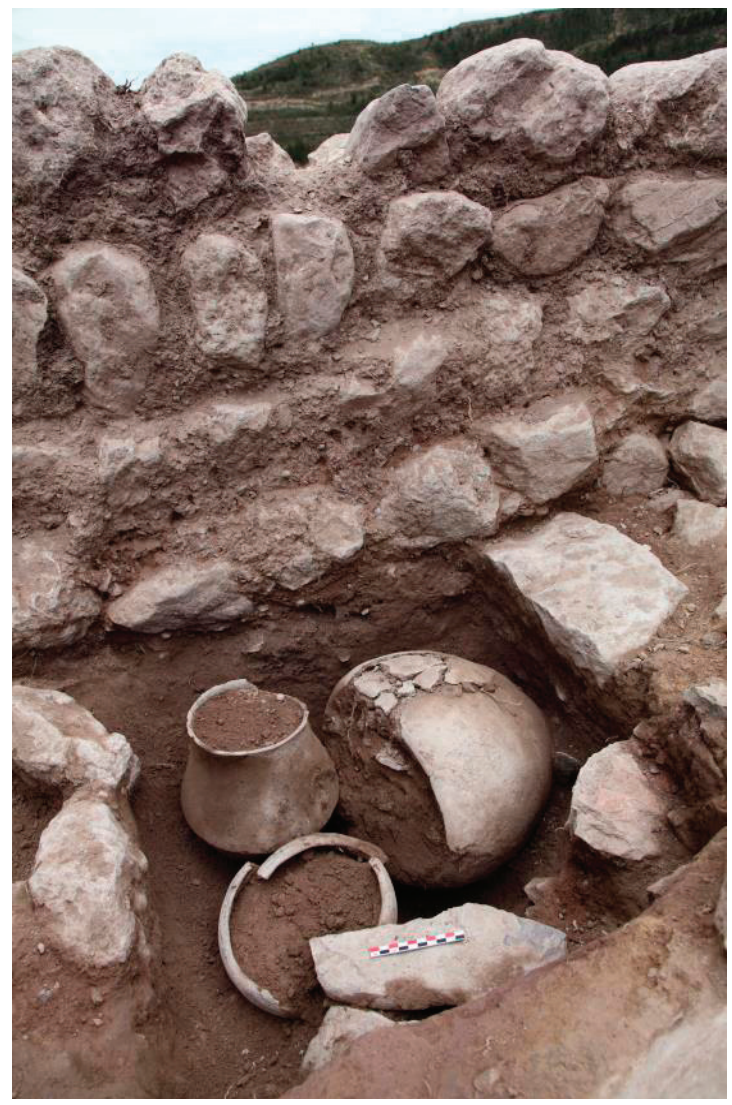

Figure 8. Burial no. 13 of La Bastida (cenotaph).

been positioned on its left side, the most common position among men.

These three spatially-related burials show different ritual transgressions in regard to the funerary treatment of sexed bodies. On one hand, the double burial included two men, and not a man and a woman. Moreover, one of them was placed according to the female custom, notwithstanding the association with a copper axe, an item exclusive to men of the intermediate class. On the other hand, burial no. 21 had a copper awl, an object reserved for women of the upper and intermediate classes, but the corpse was deposited in the typical position of men. As in the first case, the objects representative of social category followed the normal sexual ascription, but the positioning of the body did not. The presence of a cenotaph with the formal characteristics of a relatively rich infant burial between these two graves is also exceptional in the Argaric world. ${ }^{77}$

In sum, it seems that the "ritual deviations" in Room 3 were the symbolic expression of certain relationships by individuals with a distinctive gender status. The fact that these three tombs contained grave goods of a medium-high social class, and that Room 3 is one of the largest residential units $\left(90 \mathrm{~m}^{2}\right.$ preserved) and with the bigger storage capacity so far found in its neighbourhood, invites us to think about the blurred limits between transgression and privilege at the heart of class societies. If the ritual deviations detected in Room 3 represented transgressions in a not very tolerant society, it may not have been by chance that only certain groups could afford celebrating their difference. How close to a civilised society may El Argar have been if it cultivated double morals!

\section{Conclusions}

Any approach to the organization of sex and kinship relations, and to the gender symbolism in prehistoric archaeology risks being dismissed as a speculative exercise. The plentiful and varied data offered by the Argaric no doubt licences a boldness that would be out of place when dealing with the material record of other eras and places, although it would not be surprising to find even here academic taboos as rigid as those that govern many of the social relations surrounding sex and death. We have attempted to go deep into these questions through a multidimensional approach, though always with reference to the Argaric funerary data and, especially, the double burials. The analysis has sometimes been exploratory and sometimes statistical, but in each case we sought arguments for and against the hypotheses at stake. The results put forward are not conclusive, but they open new avenues of investigation and consolidate others that have been sketched in recent decades.

We have focused on sex and death as relational spheres by which Argaric social life can be gauged. Several conclusions and a diagnostic summary take shape.

1. Repression and subjection. Argaric society granted symbolic priority to blood ties to the detriment of the political unions between lineages.

a. It was a society keen to establish and remember lines of descent but which, at the same time, prescribed with utter rigidity that adults be of different sex in joint 


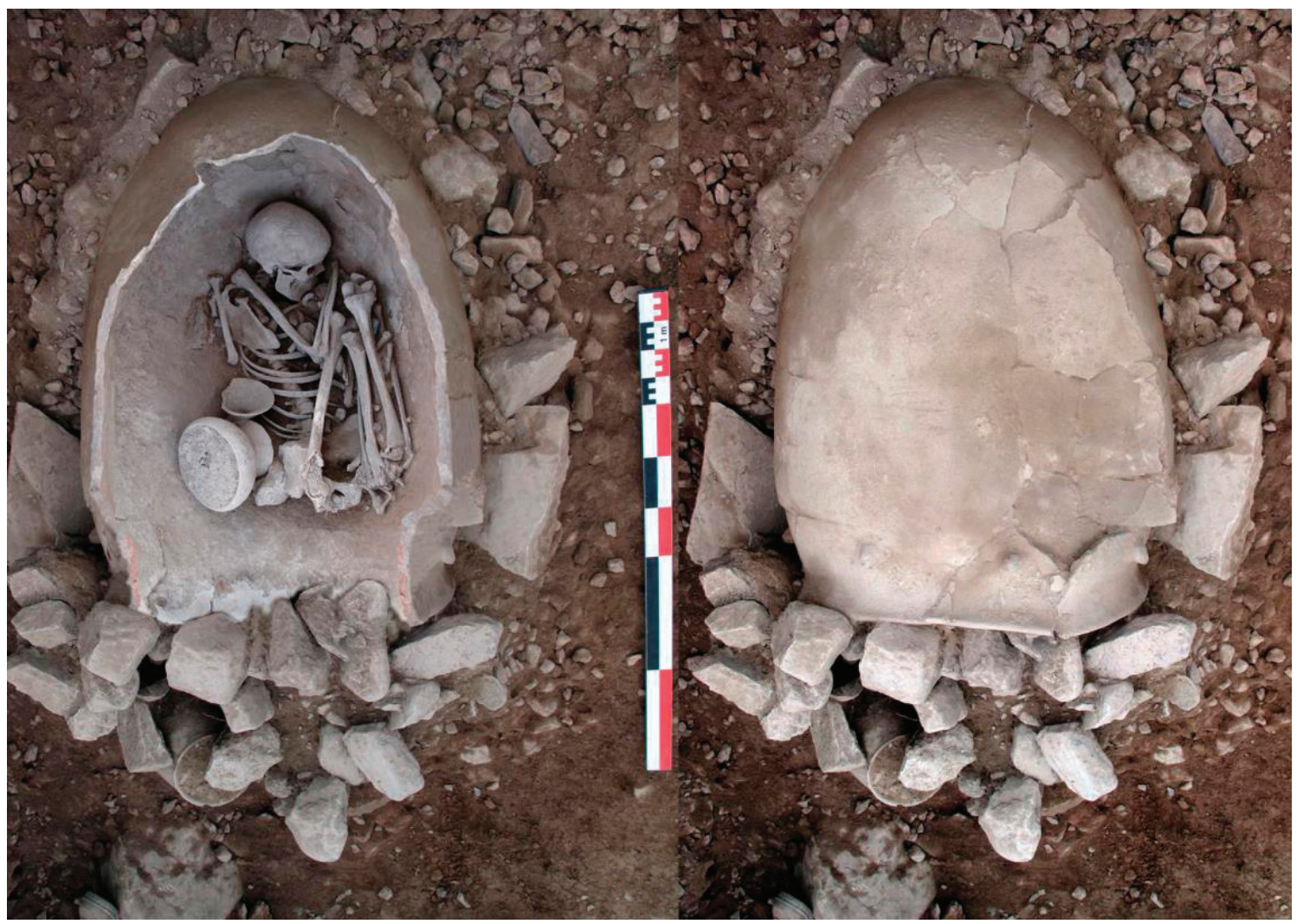

Figure 9. Burial no. 21 of La Bastida upon its discovery (right) and after the uncovering of its contents (left).

burials and upheld a binary conception that can be taken as being of heterosexual affirmation, and, therefore, of intolerance towards homosexuality. The scarcity of representations of certain alternative models of relations (male double burials) and the absence of others (female double burials) suggests a strict social control of human relationships.

b. If the predominant individual funerary rite expresses ties between certain individuals and places of residence, then double burials might symbolise the transgenerational link between those same places and specific lineages (transmission of immovable property?).

c. The concern with establishing lineages is consistent with the maintenance of property relationship, in this case the ownership of social subjects.

d. The deposition of dead bodies according to sex also followed norms, though it might have left room for the expression of gender constructions.

2. Particularism, communication and social control. El Argar society individualised death, used it to stress the continuity of genealogical ties of blood and place, and "locked it up at home" limiting the funeral to a small social circle that performed it in confined settings, away from the public eye. And yet it succeeded in spreading specific liturgies and having them respected during centuries and across $c a .35 .000 \mathrm{~km}^{2}$. Only a tight control of the ideas and ceremonies that materialise and inculcate them, orchestrated almost in a capillary level, is capable of achieving such uniformity.

3. Conservatism and permanence. Through their attachment to their places of residence and themselves, and thanks to matrilocality norms, Argaric lineages were capable of lasting and carrying on female names. 


\section{ACKNOWLEDGMENTS}

The results presented in this text were made possible by investigations funded by the Spanish Ministry of Economy and Competitiveness (project HAR2014-53860-P). We are also thankful for the support of the Council of Culture and Tourism of the Region of Murcia, as well as the cooperation of the scientific and technical staff of the "La Bastida Project" and the Research Group for Mediterranean Social Archaeoecology (ASOME) of the Autonomous University of Barcelona. Finally, we thank Miguel Valério and Bob Chapman for the English translation of this text and its revision, respectively.

\section{Notes}

1. Department of Prehistory, Autonomous University of Barcelona. Campus de Bellaterra, Edifici B. 08193 - Bellaterra (Barcelona).

2. Lull et alii (2006).

3. See the recent syntheses by Lull et alii $(2005,2009$, 2010a, b, 2013a, b, c, 2014, 2015a), Cámara and Molina (2009, 2011), Hernández Pérez et alii (2009) and Aranda et alii (2015).

4. Chapman (1991).

5. A detailed observation shows that this was not always so, because the children burials are scarcely represented (probably absent) at the formative stages of the Argaric society (Castro et alii 1993-1994, Lull et alii 2005). During these early stages, the number of tombs was very low.

6. Lull and Estévez (1986), Micó (1993), Lull et alii (2005).

7. Rojo et alii (2005).

8. Gómez Pérez et alii (2011); Liesau et alii (2015).

9. Bueno et alii (2005).

10. Bueno et alii (2005).

11. Pujante (2006), Lull et alii (2014: tabla 1).

12. Benítez de Lugo et alii (2007).

13. Ripollés (2005).

14. Ríos (2011), Gómez Pérez et alii (2011).

15. Pecero (2012).

16. Alves et alii (2010).

17. Filipe et alii (2013).

18. For the inland regions we lack quantitative data for comparison. The site of Cerro de la Encina (Granada) might be a singular case, since it is the only one in which the double burials are the main group (Aranda and Molina 2006: tab. 1).

19. They represent $58 \%$ of the total of double burials (60 cases out of 103 ).

20. According to a study of the graves of the site of El Argar, males could reach the social status of adults over the biological (osteological) age of 15 years, but with female individuals this could happen even earlier (Lull et alii 2005).

21. Buikstra et alii (1992), Castro et alii (1995).

22. $\mathrm{Chi}^{2}=25,991, \mathrm{df}=5, \mathrm{p}<, 0001$.

23. Inchaurrandieta (1870: 809), Siret and Siret (1890: 206), Cuadrado Ruiz (1947: 62), Childe (1958: 284), García Sánchez (1963: 83), Schubart et alii (1985: 95), Ayala (1991: 126), Contreras et alii (1997: 134); Arteaga (2000: 182), Schubart (2012: 42).

24. We know of only two such cases and both are double burials containing a woman and an infant: burial no. 6 of Cerro de la Encina (Sánchez Romero 2008: 14 and fig. 1) and burial no. 21 of La Almoloya (see Table 3).

25. The lavish burial no. 38 of La Almoloya (AY 38) provides a good example (Lull et alii 2015b). The woman's skeleton lay entirely over that of the man and both the taphonomic study of the articulations and 
the radiocarbon dates obtained from samples of the two individuals underscore the fact that the interment of the two bodies was very close in time (woman: MAMS-22230, 3366 \pm 32 BP; man: MAMS-22231, $3354 \pm 33 \mathrm{BP})$.

26. Castro et alii (1993-1994: 89), Lull (1997-1998: 74).

27. Cámara and Molina (2009). For the lowlands, our sample has come to comprise Fuente Álamo (Hedges et alii 1995, Van Strydonck 2005), Illeta dels Banyets (Soler et alii 2006, López Padilla et alii 2006) and Cabezo Pardo (López Padilla 2009). Various taphonomic and analytical problems have affected the dates of several double burials recently excavated at La Bastida, so that redating is needed.

28. Lull et alii (2013b). This approach is based on the comparison of the two dates available for each burial by means of Bayesian statistics (performed online through BCal at http://bcal.sheffield.ac.uk - Buck et alii 1999).

29. Personal communication from K. Alt and P. Held (University of Mainz).

30. In the context of the "Gatas" and "La Bastida" projects several lines of research aim to the recovery of ancient DNA from human bone samples. Sampling included burials from other sites, such as Fuente Álamo and Lorca. The analyses have been conducted in cooperation with the laboratories of the School of Human Evolution and Social Change - Arizona State University (Jane E. Buikstra), the Unitat d'Antropologia - Autonomous University of Barcelona (Assumpció Malgosa) Department of Genetics, Harvard Medical School (D. Reich, C. Lalueza) and of the Institut für Anthropologie - University of Mainz (K. Alt, C. Roth). The results have so far been unfruitful, because DNA preservation is usually very low and no double burial has supplied data for both occupants.

31. Conversely, an infant could be relocated in an adult grave as the second occupant. This is the case with burial no. 121 of Castellón Alto, where the skeleton of a partly mummified young man was found alongside the incomplete and not wholly skeletonized body of an infant deposited initially in a different place (Molina et alii 2003). Secondary interments might explain the cenotaphs (perhaps "emptied graves"), as well as those graves whose excavation yielded a few bone fragments of infants.

32. Sex estimation of inmature individuals from morphometric traits in the skeleton is not yet reliable enough. The estimates published in recent years for different Iberian sites are mostly based on the methods by Schutkowski (2013) and/or Loth and Henneberg (2001), dealing with small samples. Since the application of these methods in forensic collections has had little success (Coqueugniot et alii 2002, Scheuer 2002, Vlak et alii 2008), it would be preferable to dismiss the results of their use on archaeological collections.

33. Cerro de la Encina 6 and La Almoloya 21 (see above).

34. La Bastida 6 (2009 season), Castellón Alto 121, Monteagudo 3 and Fuente Álamo 89.

35. Castro et alii $(1995,1999)$.

36. Martínez Santa-Olalla (1947: 117-119 y fig. 16), Ruiz Argilés and Posac (1956), Lull et alii (2012).

37. If we take into account the contextual data offered on the association between buildings and burials by the Siret brothers (Siret and Siret 1890: lám. XV) Pedro Flores's field notes (Schubart and Ulreich 1991), and also available sex and age estimates (Jacques 1890, Kunter 1990), then both circumstances are seen also at the site of El Argar.

38. Buikstra and Hoshower (1994). This kind of craniometric analysis assumes that the gender with greater variability is also the most mobile and, therefore, the one with a wider geographical distribution owing to a post-marital shift of residence (Konigsberg 1988).

39. Díaz-Zorita et alii (2011: graphs 1 and 2).

40. K. Alt (pers. comm., 2013).

41. Castro et alii (1993-1994: 89), Lull (1997-1998: 74).

42. Hauser and de Stephano (1989), Turner et alii (1991).

43. Lull et alii (2004).

44. We have considered the burials in Table 2, with the exception of those that contained at least one infant, as well as nine cases in which data on the composition of the grave goods is lacking, doubtful or incomplete (four from Baeza, five from Castellón Alto, one from Illeta dels Banyets and another from Cabezo Pardo).

45. Sanahuja (2002). 
46. See e.g. Picazo (1997), Alarcón (2007), González Marcén et alii (2008), Alarcón and Sánchez Romero (2010). It must be noted that "maintenance activities" are not to be confused with "maintenance production", a category referring strictly to the moment of production in the social life of both objects and subjects, which involved a very different ontological and methodological approach (Castro et alii 1998).

47. The term "dailiness" often appears alongside "maintenance activities" and presents the same ambiguity.

48. Lull (2007a: 14-17).

49. Lull (2007b).

50. In the skeletons of adults, the secondary sexual traits usually allow us to distinguish between male and female individuals. In Spanish, the category "alofiso" refers to unclassifiable cases and stresses, in osteological terms, the degree of overlap in all human populations.

51. Among the items included in the category of weaponry, only the axes are not necessarily specialised in the exercise of violence.

52. Siret and Siret (1890), Lull (1983), Lull and Estévez (1986).

53. Castro et alii (1993-1994).

54. Siret and Siret (1890), Lull and Estévez (1986), González Marcén (1991, 1994), Micó (1993), Castro et alii (1993-1994).

55. Castro et alii (1993-1994).

56. Montón (2010).

57. Risch (2002: 275).

58. This fact has already been noticed in several burials or single sites (Kunter 2000: 269; Schubart 2004: 68-77; López Padilla et alii 2006: 159; Rihuete et alii 2011: 63; Sánchez and Alarcón 2012: 69).

59. It must be noted that the series from the province of Murcia represents $55 \%$ of the sample and includes unpublished data from recent excavations in three sites (La Bastida, La Almoloya and Monteagudo).

60. The male group includes 57 skeletons in strict position and 5 more lying on the back but with the lower extremities flexed and turned laterally. In the female group we have 55 skeletons in strict lateral position and 13 on the back. Bodies facing down (lying on the chest, i.e. prone position) are very rare; we just know five cases, all of them female -La Bastida 77 (2009-2012 campaigns), Cerro de la Virgen 14 (Schüle 1980: taf. 117a), Monteagudo 2 (unpublished series, J. A. Zapata), and La Almoloya 37 and 47 (2014 campaign)-, and in all five the lower extremities are flexed and turned to the right side of the body.

61. $\mathrm{Chi}^{2} 47,034, \mathrm{df}=1, \mathrm{p}<, 0001$.

62. Cerro de la Encina 8 and Fuente Alamo 90, respectively.

63. With the possible exception of Cerro de la Encina 15, a man without grave goods dated in the Post-Argaric Bronze Age according to Beta-230010: $3140 \pm 40$ bp (Aranda et alii 2008: tab. 1).

64. Häusler (1991, 1994).

65. Primas (1977), Salanova (2011).

66. Esparza et alii (2012), Nájera et alii (2010), Soriano (2010), Gómez Pérez et alii (2011), Ríos (2011), Hunt (2012), Pavón (2008).

67. Pecero (2012).

68. Schubart (2012: 64-68).

69. The inclusion of burial no. 80 is the result of a lapse. In the same publication, Schubart (2012: 143) specifies that the skeleton resting on the right side was the female one.

70. Another double burial with two men has been found at Eras del Alcázar (burial no. 9) (Lizcano et alii 2009: 39; Nocete et alii 2010: tabla 5), a third one appeared at the site of Cerro del Alcázar (again in the province of Jaén) (burial no. 12) (Robledo y Trancho 2003: 7), and a possible fourth example at Cerro de la Encantada (Ciudad Real) (burial no. 37) (Lapuente 2008: tabla 6).

71. $\mathrm{M}^{\mathrm{a}}$ Inés Fregeiro and Camila Oliart (ASOME research group - Autonomous University of Barcelona) collaborate in the anthropological study of the burials of La Bastida. The second occupant was buried over the first, before the latter was fully skeletonised. The C14 analysis of bone samples has not produced reliable results due to the lack of collagen, whereas the date of seeds of barley deposited as grave goods falls between the $18^{\text {th }}$ and $17^{\text {th }}$ centuries cal BCE (KIA-40099: 3405 \pm 20 BP). 
72. The investigation of the faunal remains of La Bastida is undertaken by Lourdes Andúgar (ASOME research group - Autonomous University of Barcelona).

73. Hans Peter Stika (University of Höhenheim, Stuttgart) is responsible for the identification of the carpological remains of La Bastida.

74. We refer to the case of the Egyptian tomb of Niankhkhnum and Khnumhotep (Fifth Dynasty). Most researchers opted for interpreting it as the tomb of two brothers, until Reeder (2000) presented convincing arguments in favour of the view that the two men were lovers, one of them having been represented with the iconographic traits normally applied to a wife.

75. Type 2B3y, according to Lull (1983).

76. The connection between burial jars of the Type 2B3y and infant burials is well-known. The preference for this ceramic subtype as a funerary container for very young infants as well as the suitability of the vessel's size for the body's corporal volume was observed at Gatas (Buikstra et alii 1992: 273-274). The use of Type 2 containers for individuals deceased before the age of 3 years at the necropolis of El Argar has also been observed (Lull et alii 2005).

77. To the examples of La Bastida (Lull et alii 2012: 64), we must add those of El Rincón de Almendricos (Martínez Rodríguez et alii 1996: note 58; Ayala 2003: 206), Cerro de las Viñas (Martínez Rodríguez et alii 1996: note 58) and Lorca, Zapatería St. no. 11 (Martínez 1995: 74). In the province of Almería we know of the cenotaphs of Gatas (burial no. 27 - Buikstra et alii 1992: 264) and Fuente Álamo (burial no. 78 - Schubart 2013: 142), and we could perhaps add burials nos. 326, 328, 357, 551, 680 and 879 of El Argar if we interpret correctly the data collected from Pedro Flores field notes.

\section{REFERENCES}

Al-Oumaoui, I. (2009): Afinidades entre poblaciones antiguas de la península Ibérica. Antropología dental. Ph.D. dissertation: University of Granada.

Alarcón, E. (2007): Las prácticas de cuidados en las sociedades prehistóricas: la cultura argárica, Arqueología y Territorio, 4, pp. 233-249.

Alarcón, E. (2010): Continuidad y cambio social. Las actividades de mantenimiento en el poblado argárico de Peñalosa (Baños de la Encina, Jaén). Ph.D. dissertation: University of Granada.

Alarcón, E. (2012): Teoría y método: arqueología de mujeres y las relaciones de género en la Edad del Bronce del sureste de la península Ibérica, Arkeogazte, 2: 33-55.

Alarcón, E., SÁnchez Romero, M. (2010): Maintenance activities as a category for analysing Prehistoric Societies, in DOMMASNES, L.H., HJØRUNGDAL, T., MONTÓN, S., SÁNCHEZ ROMERO, M., WICKER, L. (eds.), Situating gender in European Archaeologies. Archaeolingua Foundation, Budapest: 261-282.

Alonso, C. (2013): Las tumbas campaniformes del monumento funerario "El Hundido" (Monasterio de Rodilla, Burgos), Munibe, 64: 89-103.

Alves, C., Costeira, C., Estrela, S., Porfírio, E., Serra, M., Soares, A.M., Moreno-García, M. (2010): Hipogeus funerários do Bronze pleno da Torre Velha 3 (Serpa, Portugal). O Sudeste no Sudoeste?!, Zephyrus, LXVI, (2): 133-153.

Andrés, Ma T., García García, Mª́., Sesma, J. (2007): Tres Montes. Un sepulcro singular del III milenio en las Bardenas Reales, La Tierra te sea leve. La arqueología de la muerte en Navarra (catálogo de la exposición 27 de noviembre de 2007 - 30 de abril de 2008 en el Museo de Navarra). Gobierno de Navarra, Pamplona: 84-88.

Aranda, G. (2011): Nuevos actores para viejos escenarios. La sociedad argárica, en Memorial Siret. I Congreso de Prehistoria de Andalucía. La tutela del patrimonio prehistórico. Junta de Andalucía, Sevilla: 249-270.

Aranda, G.,Alarcón, E., Murillo-Barroso, M., Montero, I., Jiménez-Brobeil, S., SÁnchez Romero, M., RodríGuez ArizA, Mª̂. (2012): El yacimiento argárico del Cerro de San Cristóbal (Ogíjares, Granada), Menga, 3: 141-164. 
Aranda. G., Molina, F. (2005): Intervenciones arqueológicas en el yacimiento de la Edad del Bronce del Cerro de la Encina (Monachil, Granada), Trabajos de Prehistoria, 62 (1): 165-179.

Aranda. G., Molina, F. (2006): Wealth and power in the Bronze Age of the South-East of the Iberian Peninsula: the funerary record of Cerro de la Encina, Oxford Journal of Archaeology, 25 (1): 47-59.

Aranda, G., Molina, F., Fernández, S., SÁnchez, M., Al-Oumaoui, I., JimÉnez-Brobeil, S., Roca, M. (2008): El poblado y necrópolis argáricos del Cerro de la Encina (Monachil, Granada). Las campañas de excavación de 2003-05, Cuadernos de Prehistoria y Arqueología de Granada, 18: 219-264.

Aranda, G., Montón-Subías, S., Sánchez-Romero, M. (2015): The Archaeology of Bronze Age Iberia: Argaric Societies. Routledge, New York.

Arteaga, O. (2000): La sociedad clasista inicial y el origen del Estado en el territorio de El Argar, Revista Atlántica-Mediterránea de Prehistoria y Arqueología Social, 3: 121-219.

Ayala, MaM. (1991): El poblamiento argárico en Lorca. Estado de la cuestión. Real Academia Alfonso X El Sabio, Murcia.

Ayala, M M. (2003): Poblados de llanura y poblados de altura de la Edad del Bronce en la cultura de El Argar, in Ramallo, S. F. (ed.), Estudios de arqueología dedicados a la profesora Ana María Muñoz Amilibia. University of Murcia: 175-218.

Ayala, MaaM., Jiménez, S., Malgosa, A., Alesan, A., Safont, S. (1999): Los enterramientos infantiles en la prehistoria reciente del levante y sureste peninsular, Anales de Prehistoria y Arqueología de la Universidad de Murcia, 15: 15-27.

Ayala MaM., Tudela, MáL. (1993): La espada del poblado argárico "La Cabeza Gorda o Cabezo de la Cruz". Totana (Murcia), Verdolay, 5: 17-23.

Benítez, L., Álvarez, H.J., Moraleda, J., Molina, M. (2007): Consideraciones acerca del Bronce de La Mancha a partir de la investigación de la cueva prehistórica fortificada de Castillejo del Bonete (Terrinches, Ciudad Real). Campañas 2003-2005, in Actas de las I Jornadas de Arqueología de Castilla-La Mancha (Cuenca, 2005). University of Castilla-La Mancha. Cuenca: 231-262.

Blasco, C., Liesau, C., Delibes, G., Baquedano, E., Rodríguez Cifuentes, M. (2005): Enterramientos campaniformes en ambiente doméstico: el yacimiento de Camino de las Yeseras (San Fernando de Henares, Madrid), in Rojo, M., Garrido, R., García Martínez de Lagrán, Í. (eds.), El Campaniforme en la Península Ibérica y su contexto europeo. University of Valladolid - Junta de Castilla y León: 457-479.

Buck C.E., Christen J.A., James G.N. (1999): BCal: an on- line Bayesian radiocarbon calibration tool, Internet Archaeology, 7.

Bueno, P., Barroso, R., Balbín, R. (2005): "Ritual campaniforme, ritual colectivo: la necrópolis de cuevas artificiales del Valle de las Higueras (Huecas, Toledo)", Trabajos de Prehistoria, 62 (2): 67-90.

Buikstra, J.E., Castro, P., Chapman, R., González Marcén, P., Hoshower, L., Lull, V., Risch, R., Sanahuja, MáE. (1992): La necrópolis de Gatas, Anuario Arqueológico de Andalucía, 1990: 261-276.

Buikstra, J.E., Hoshower, L. (1994): Análisis de los Restos Humanos de la necrópolis de Gatas, In CaStro, P., Chapman, R., Colomer, E., Gili, S., González Marcén, P., Lull, V., Micó, R., Montón, S., Rinuete, C., Risch, R., Ruiz, M., Tenas, M. (eds.), Proyecto Gatas: Sociedad y economía en el sudeste de España c. 2500-900 cal ANE. Junta de Andalucía, Sevilla: 339-398.

CÁmara, J.A. (2001): El ritual funerario en la Prehistoria Reciente en el Sur de la Península Ibérica. British Archaeological Reports, International Series, 913, Oxford.

CÁmara, J.A., Molina, F. (2009): El análisis de la ideología de emulación: el caso de El Argar, Cuadernos de Prehistoria y Arqueología de la Universidad de Granada, 19: 163-194.

CÁmara, J.A., Molina, F. (2010): Relaciones de clase e identidad en El Argar. Evolución social y segregación espacial en los Altiplanos granadinos (c. 2000-1300 cal AC), Arqueología Espacial, 28: 21-40.

CÁmara, J.A., Molina, F. (2011): Jerarquización social en el mundo Argárico (2000-1300 aC), Quaderns de Prehistòria i Arqueologia de Castelló, 29: 77-104.

Castro, P., Chapman, R., Colomer, E., Gili, S., González Marcén, P., Lull, V., Micó, R., Montón, S., Rihuete, C., Risch, R., Ruiz Parra, M., Sanahuja, MaEE., Tenas, M. (1994): Proyecto Gatas: Sociedad y Economía en el sudeste de España c. 2500-900 cal ANE. Junta de Andalucía, Sevilla. 
Castro, P., Chapman, R.w., Gili, S., Lull, V., Micó, R., Rihuete, C., Risch, R., Sanahuja, MªE., (19931994): Tiempos sociales de los contextos funerarios argáricos, Anales de Prehistoria de la Universidad de Murcia, 9-10: 77-107.

Castro, P., Chapman, R., González Marcén, P., Lull, V., Micó, R., Picazo, M., Risch, R., Sanahuja, $M^{a}$ E. (1993): 4a Campaña de excavaciones en el yacimiento de Gatas (Turre - Almería). Septiembre 1991, Anuario Arqueológico de Andalucía, 1991: 17-23.

Castro, P., Chapman, R., Escoriza, T., Gili, S., Lull, V., Micó, R., Risch, R., Rihuete, C., Sanahuja, $M^{a} E$. (1999): $5^{\text {a }}$ Campaña de Excavaciones en el yacimiento de Gatas (Turre, Almería). 1995, Anuario Arqueológico de Anadalucía, 1995: 7-14.

Castro, P., Chapman, R., Escoriza, T., Lull, V., Micó, R., Rihuete, C., Risch, R., Sanahuja, MãE. (2004): Sexta campaña de excavaciones en Gatas. La meseta superior del Cerro de los Castellones, Anuario Arqueológico de Anadalucía, 2001: 9-14.

Castro, P., Lull, V., Micó, R., Rinuete, C. (1995): La Prehistoria Reciente en el sudeste de la Península Ibérica. Dimensión socio-econoómica de las prácticas funerarias, in FÁBREGAS, R., PÉREZ, F., FERNÁNDEZ, C. (eds.), Arqueoloxía da Morte na Península Ibérica desde as Orixes ata o Medievo. University of Vigo, Xinzo de Limia: 129-167.

Contreras, F. Cámara, J.A., Robledo, B., Trancho, G.J. (2001): La Necrópolis, in Contreras, F. (coord.), Proyecto Peñalosa. Análisis histórico de las comunidades de la Edad del Bronce del Piedemonte meridional de Sierra Morena y Depresión Linares-Bailén. Arqueología Monografías 10. Junta de Andalucía, Sevilla: 287-324.

Contreras, F., Rodríguez Ariza, MåO., Cámara, J.A., Moreno, A. (eds.) (1997): Hace 4000 años. Vida y muerte en dos poblados de la Alta Andalucía. Empresa Pública de Gestión de Programas Culturales, Sevilla.

Coqueugniot, H., Giacobini, G., Malerba, G. (2002): L'utilisation de caracteres morphologiques dans la diagnose sexuelle des mandibules d'enfants: application à la collection ostéologique de Turin (Italie), Bulletin et Mémoirs de la Socieété d'Anthropologie de Paris, 14 (1-2): 131-139.

Cuadrado, E. (1945): La Almoloya, nuevo poblado de la cultura de El Argar, Anales de la Universidad de Murcia, Letras, 3: 355-382.

Cuadrado, J. (1947): Algunos yacimientos prehistóricos de la zona Totana-Lorca, Boletín Arqueológico del Sudeste Español, 3: 56-65.

Childe, V. G. (1958): The Dawn of European Civilization (6th ed.). Alfred Knopf, New York.

Delgado RaAcK, S. (2013): Tecnotipología y distribución especial del material macrolítico del Cerro de la Virgen de Orce (Granada). British Archaeological Reports, Int. Ser. S2518, Oxford.

Díaz-Zorita, M., Prevedorou, E.A., Buikstra, J.E., Knudson, K.J., Gordon, G., Anbar, A. (2011): Movilidad y paleodieta en la comunidad argárica de Gatas: análisis de ${ }^{87} \mathrm{Sr} r{ }^{86} \mathrm{Sr}$, $\mathrm{d}^{18} \mathrm{O}$ y d ${ }^{13} \mathrm{C}$, en Memorial Luis Siret. I Congreso de Prehistoria de Andalucía. La tutela del patrimonio prehistórico. Junta de Andalucía, Sevilla: 603-606.

EIROA, J.J. (1993-1994): Aspectos funerarios del poblado de Bajil (Moratalla, Murcia, Anales de Prehistoria y Arqueología, 9-10: 55-76.

ELLER, C. (2000): The myth of matriarchal prehistory. Why an invented past won't give women a future. Beacon Press, Boston.

Esparza, A., Velasco, X., Delibes, G. (2012): HUM2005-00139: Planteamiento y primeros resultados de un proyecto de investigación sobre la muerte en Cogotas I, in Rodríguez Marcos, J. A., Fernández Manzano, J. (eds.), Cogotas I. Una cultura de la Edad del Bronce en la Península Ibérica. University of Valladolid, Valladolid: 259-320.

Fabián, J.F., Blanco, A. (2012): Cuatro enterramientos calcolíticos en hoyo del Cerro de la Cabeza (Ávila), Complutum, 23 (1): 99-120.

Filipe, V., Godinho, R., Granja, R., Ribeiro, A., Valera, A. C. (2013): Bronze Age funerary spaces in Outeiro Alto 2 (Brinches, Serpa, Portugal): the hypogea cemetery, Zephyrus, LXXI (1): 107-129.

Gallardo, J., González Ballesteros, J.A., (2006): Un enterramiento en cista de mampostería hallado en el cerro del Castillo de Lorca, Alberca, 4: 51-59. 
García Martínez, M. (1999): Totana en imágenes. Ayuntamiento de Totana, Totana.

García Puchol, O., Gómez Pérez, O., Iborra, P. (2011): Sepulturas y depósitos especiales, in Pérez Jordá, G., Bernabeu, J., Carrión, Y., García Puchol, O. Molina, L., Gómez Puche, M. (eds.), La Vital (Gandía, Valencia). Vida y muerte en la desembocadura del Serpis durante el III y el I milenio a.C. Prehistory Museum of Valencia -Trabajos Varios n ${ }^{\circ}$ 113, Valencia: 83-96.

García SÁnchez, M. (1963): El poblado de El Cerro del Culantrillo, en Gorafe (Granada), Archivo de Prehistoria Levantina, X: 69-96.

GARCíA SANDOVAL, E. (1964): Segunda campaña de excavaciones arqueológicas en el yacimiento argárico de “El Puntarrón Chico", Beniaján (Murcia), Noticiario Arqueológico Hispánico, VI, 1-3: 108-114.

Garrido, R., Rojo, M., García Martínez De Lagrán, Í. (2005): El Campaniforme en la Meseta central de la Península Ibérica, in Rojo, M., Garrido, R., García Martínez de Lagrán, Í. (eds.), El Campaniforme en la Península Ibérica y su contexto europeo. University of Valladolid-Junta de Castilla y León: 411-435.

Gómez Pérez, J.L., Blasco, C., Trancho, G., Grueso, Í., Ríos, P., Martínez-Ávila, M. (2011): Los protagonistas, in Blasco, C., Liesau, C., Ríos, P. (eds.), Yacimientos calcoliticos con campaniforme de la Región de Madrid: nuevos estudios. Patrimonio Arqueológico de Madrid, 6, Autonomous University of Madrid, Madrid: 101-132.

GONZÁLEZ MARCÉN, P. (1991): Cronología del grupo argárico. Ensayo de fasificación radiométrica a partir de la curva de calibración de alta precisión. Ph.D. dissertation: Autonomous University of Barcelona, Bellaterra.

GonzÁlez Marcén, P., (1994): Cronología del grupo argárico, Revista d'Arqueologia de Ponent, 4: 7-46.

González Marcén, P., Montón, S., Picazo, M. (2008): Towards an archaeology of maintenance activities, in Montón, S., SÁnchez Romero, M. (eds.), Engendering Social Dynamics: the Archaeology of Maintenance Activities. British Archaeological Reports, Int. Ser. S1862, Oxford: 3-8.

Haro, M. (2012): La Puesta en Valor de yacimientos arqueológicos de la Prehistoria Reciente en el sur de la Península Ibérica. Ph.D. dissertation: University of Granada.

Hauser, G. y Stephano, G.F. de (1989): Epigenetic Variants of the Human Skull, Schweizerbart, Stuttgart.

HäUSLER, A. (1991): Geschlechtsdifferenzierte Bestattungssitten im Neolithikum und in der frühen Bronzezeit Mitteleuropas, Saeculum, 41: 332-348.

HäUsLeR, A. (1994): Grab- und Bestattungssitten des Neolithikums und der frühen Bronzezeit in Mitteleuropa, Zeitschrift für Archäologie, 28: 23-61.

Hedges, R.E.M., Housley, R.A., Ramsey, C.B., Klinken, G.J. (1995): Radiocarbon dates from the Oxford AMS System: Archaeometry Datelist 19, Archaeometry 37 (1): 195-214.

Hernández Pérez, M., Soler, J., LóPez Padilla, J.A. (eds.) (2009): En los confines del Argar. Una cultura de la Edad del Bronce en Alicante en el centenario de Julio Furgús. Archaeological Museum of Alicante, Alicante.

Hunt, M. (2012): Intervenciones arqueológicas en el área del Proyecto Minero Cobre Las Cruces (19962011). De la Prehistoria a la Época Contemporánea. Fundación Cobre Las Cruces, Sevilla.

InCHAURRANDieTA, R. (1870): "Estudios Pre-Históricos. La Edad del Bronce en la prov. de Murcia". Boletín-Revista de la Universidad de Madrid, II, 13: 806-815.

JACQues. V. (1890): Etnología, in SIRET, H., SIRET, L., Las Primeras Edades del Metal en el Sudeste de España. Barcelona: 336-484.

JimÉnez Brobeil, S., García SÁncheZ, M. (1989-90): Estudio de los restos humanos de la Edad del Bronce del Cerro de la Encina (Monachil, Granada), Cuadernos de Prehistoria de la Universidad de Granada, 14-15: 157-180.

Konigsberg, L.W., (1988): Migration models of prehistoric postmarital residence, American Journal of Physical Anthropology, 77 (4): 471-482.

Kunter, M. (1990): Menschliche Skelettreste aus Siedlung der El Argar-Kultur. Madrider Beiträge, 18, Deutsches Archäologisches Institut Madrid-Philipp von Zabern, Mainz.

KunTer, M. (2000): Los restos de esqueletos humanos hallados en Fuente Álamo durante las campañas de 1985, 1988 y 1991, in Schubart, H., Pingel, V., Arteaga, O. (eds.) (2000), Fuente Álamo. Las excavaciones arqueológicas 1977-1991 en el poblado de la Edad del Bronce. Junta de Andalucía, Sevilla: 265-282. 
LAPUENTE, M. (2008): EL conocimiento de las poblaciones del pasado a través de los restos óseos: Características Biológicas de la Población de La Encantada (Granátula de Calatrava, Ciudad Real, II milenio a.C). Final Project of Degree, Autonomous University of Madrid.

Liesau, C., Blasco, C., Ríos, P., Flores, R. (2015): La mujer en el registro funerario campaniforme y su reconocimiento social, Trabajos de Prehistoria, 72 (1): 105-125.

Lizcano, R., Nocete, F., Peramo, A., (2009): Las Eras. Proyecto de puesta en valor y uso social del patrimonio arqueológico de Úbeda (Jaén). University of Huelva, Huelva.

López Padilla, J.A. (2009): Cabezo Pardo (San Isidro/Granja de Rocamora), in Hernández Pérez, M.s., Soler, J., López Padilla, J.A. (eds.), En los confines del Argar. Una cultura de la Edad del Bronce en Alicante. Archaeological Museum of Alicante, Alicante: 156-159.

López Padilla, J.A., Belmonte, D., De Miguel, P. (2006): Los enterramientos argáricos de la Illeta dels Banyets de El Campello, Alicante. Prácticas funerarias en la frontera oriental de El Argar, in SOLER, J. (ed.) (2006), La ocupación Prehistórica de la Illeta dels Banyets (El Campello, Alicante. Serie Mayor 5, Diputación de Alicante/MARQ, Alicante: 119-172.

Loth, S., Henneberg, M. (2001): Sexually dimorphic mandibular morphology in the first few years of life, American Journal of physical anthropology, 115: 179-186.

Lull, V. (1983): La cultura de El Argar. Un modelo para el estudio de las formaciones económico-sociales prehistóricas. Akal, Madrid.

Lull, V. (1997-1998): El Argar: la muerte en casa, Anales de Prehistoria y Arqueología de la Universidad de Murcia, 13-14: 65-80.

Lull, V. (2007a): Ética y Arqueología, Trabajos de Prehistoria, 64 (1): 13-21.

Lull, V. (2007b): Los objetos distinguidos. La arqueologia como excusa. Bellaterra. Barcelona.

Lull, V., Estévez, J. (1986): Propuesta metodológica para el estudio de las necrópolis argáricas, en Homenaje a Luis Siret (1934-1984). Junta de Andalucía, Sevilla: 441-452.

Lull, V., Micó, R., Rinuete, C., Risch, R. (2005): Property Relations in the Bronze Age of South-western Europe: an Archaeological Analysis of Infant Burials from El Argar (Almeria, Spain), Proceedings of the Prehistoric Society, 71: 247-268.

Lull, V., Micó, R., Rinuete, C., Risch, R. (2006): La investigación de la violencia: una aproximación desde la arqueología, Cypsela, 16: 87-108.

Lull, V., Micó, R., Rihuete, C., Risch, R. (2009): El Argar: la formación de una sociedad de clases, in Hernández Pérez, M.s., Soler, J., LóPez Padilla, J.A. (eds.), En los confines del Argar: una cultura del Bronce en Alicante en el centenario de Julio Furgús. Archaeological Museum of Alicante, Alicante: 224245.

Lull, V., Micó, R., Rihuete, C., Risch, R. (2010a): Las relaciones políticas y económicas de El Argar, Menga, 1: 11-35.

Lull, V., Micó, R., Rinuete, C., Risch, R. (2010b): Metal and social relations of production in the 3rd and 2nd millenia BC in the southeast of the Iberian Peninsula, Trabajos de Prehistoria, 67 (2): 323-347.

Lull, V., Micó, R., Rihuete, C., Risch, R. (2012): "Proyecto La Bastida": economía, urbanismo y territorio de una capital argárica", Verdolay, 13: 57-70.

Lull, V., Micó, R., Rinuete, C., Risch, R. (2013a): Political collapse and social change at the end of El Argar, in Meller, H., Bertemes, F., Bork, H.-R., Risch, R. (eds.), 1600 Cultural change in the shadow of the Thera-Eruption? Landesmuseum für Vorgeschichte von Sachsen-Anhalt, 9, Halle: 283-302.

Lull, V., Micó, R., Rinuete, C., Risch, R. (2013b): Funerary practices and kinship in an Early Bronze Age society: a Bayesian approach applied to the radiocarbon dating of Argaric double tombs, Journal of Archaeological Science, 40: 4626-4634.

Lull, V., Micó, R., Rinuete, C., Risch, R. (2013c): Bronze Age Iberia, in HARDING, A., FOKKENS, H., The Oxford Handbook of the European Bronze Age. Oxford University Press, Oxford: 594-616.

Lull, V., Micó, R., Rinuete, C., Risch, R. (2014): The social value of silver in El Argar, Tagungen des Landesmuseums für Vorgeschichte Halle, 11-II: 557-576.

Lull, V., Micó, R., Rinuete, C., Risch, R. (2015a): Transition and conflict at the end of the 3rd millenium BC in Southern Iberia, in Meller, H., Arz, H. W., Jung, R., Risch, R. (eds.), 2200 BC-Ein Klimasturz als 
Ursache für den Zerfall der Alten Welt? Landesamt für Denkmalpflege und Archäologie Sachsen-Anhalt, Halle: 365-407.

Lull, V., Micó, R., Rinuete, C., Risch, R. (2015b): La Almoloya. Premier palais de l'âge du Bronze occidental, Archéologia, 530: 58-63.

Malgosa, A., Alesan, A., Safont, S., Ballbé, M., Ayala, MªM. (2004): A Dystocic childbirth in the Spanish Bronze Age, Internacional Journal of Osteoarchaeology, 14: 98-103.

Martínez Rodríguez, A. (1990): Aportaciones a la secuencia histórica de la ciudad de Lorca, en Lorca. Pasado y presente. Aportaciones a la historia de la región de Murcia, Vol. 1., Lorca Council/CAM, Lorca: 71-86.

Martínez RodríGuez, A. (1995): I Fase de excavaciones de urgencia en la calle Zapatería no 11 (Lorca), Memorias de Arqueología, 3: 64-80.

Martínez RodríGuez, A., Ponce, J. (2002a) Excavación arqueológica de urgencia en el subsuelo de la antigua iglesia del convento de las Madres Mercedarias (C/Zapatería y C/ Cava, Lorca)", Memorias de Arqueología, 10: 90-137.

Martínez Rodríguez, A., Ponce, J. (2002b): Segunda intervención arqueológica en la Plaza de Juan Moreno, $\mathrm{n}^{\mathrm{o}}$ 8, confluencia con calle los Tintes, Lorca, Memorias de Arqueología, 10: 150-160.

Martínez Rodríguez, A., Ponce, J., Ayala, Ma M. (1996): Las prácticas funerarias de la cultura argárica en Lorca. Ayuntamiento de Lorca, Lorca.

Martínez Rodríguez, A., Ponce, J., Ayala, Ma M. (1999): Excavaciones de urgencia del poblado argárico de Los Cipreses, Lorca. Años 1992-1993, Memorias de Arqueología, 8: 155-182.

Molina., F., Aguayo, P., Fresneda, E., Contreras, F. (1986): Nuevas investigaciones en yacimientos de la Edad del Bronce en Granada, en Homenaje a Luis Siret (1934-1984). Junta de Andalucía, Sevilla: 353-360.

Molina, F., CÁmara, J.A. (2009): La cultura argárica en Granada y Jaén, en En los Confines del Argar. Una cultura de la Edad del Bronce en Alicante. Museo Arqueológico de Alicante: 196-223.

Molina, F., Rodríguez-Ariza, Ma O., JimÉnez, S., Botella, M. (2003): La sepultura 121 del yacimiento argárico de El Castellón Alto (Galera, Granada), Trabajos de Prehistoria, 60 (1): 153-158.

Montón, S. (2010): Muerte e identidad femenina en el mundo argárico, Trabajos de Prehistoria, 67 (1): 119-137.

Nájera, T., Molina, F., Jiménez-Brobeil, S., Sánchez Romero, M., Al-Oumaoui, I., Aranda, G., Delgado, A., LAFFRANCHI, Z. (2010): La población infantil de la Motilla del Azuer: Un estudio bioarqueológico, Complutum, 21 (2): 69-102.

Nocete, F., Lizcano, R., Peramo, A., Gómez, E. (2010): Emergence, collapse and continuity of the first political system in the Guadalquivir Basin from the fourth to the second millennium BC: The long-term sequence of Úbeda (Spain), Journal of Archaeological Science, 29: 219-237.

Pavón, I. (2008): El mundo funerario de la Edad del Bronce en la Tierra de Barros: una aproximación desde la bio-arqueología de Las Minitas. Memorias de Arqueología Extremeña, 9, Junta de Extremadura, Mérida.

Pecero, J. C. (2012): Caracterización Bioarqueológica de los restos humanos prehistóricos del proyecto minero Cobre Las Cruces, in Hunt, M., Intervenciones arqueológicas en el área del Proyecto Minero Cobre Las Cruces (1996-2011). De la Prehistoria a la Época Contemporánea. Fundación Cobre Las Cruces, Sevilla: 56-64.

Pérez Richard, E. (2007): Una cista argárica en el Castillo de Lorca, Alberca, 5: 43-52.

Pericot, L. (1934): Historia de España. Gran Historia General de los Pueblos Hispanos. Tomo I: Épocas Primitiva y Romana. Instituto Gallach, Barcelona.

PICAzo, M. (1997): Hearth and home: the timing of maintenance activities, en MOORE, J., SCOTT, E. (eds.), Invisible People and Processes, Leicester University Press: 59-67.

Primas, M. (1977): Untersuchungen zu den Bestattungssitten der ausgehenden Kupfer- und frühen Bronzezeit, Berichte der RGK, 58: 4-160.

PujAnte, A. (2003): Excavación arqueológica en el Convento de Madres Mercedarias de Lorca, ArqueoMurcia, 1: 1-67. 
Pujante, A. (2006): El yacimiento prehistórico de Los Molinos de Papel (Caravaca de la Cruz, Murcia). Intervención arqueológica vinculada a las obras de infraestructura del Plan Parcial SCR2, 1999-2000, Memorias de Arqueología, 14: 133-172.

Pujante, A., Martínez Rodríguez, A. (2010): Los enterramientos argáricos de la excavación arqueológica en el convento de Madres Mercedarias de Lorca (Murcia), Alberca, 8: 7-40.

REEDER, G. (2000): Same-sex desire, conjugal constructs, and the tomb of Niankhkhnum and Khnumhotep, World Archaeology, 32 (2): 193-208.

Rinuete, C., Oliart, C., Fregeiro, Maa I. (2011): Algo más que huesos. Aproximación a la población argárica a la luz de los enterramientos del convento de Madres Mercedarias de Lorca (Murcia), Alberca, 9: 39-79.

Ríos, P. (2011): Territorio y sociedad en la Región de Madrid durante el III milenio a.C. El referente del yacimiento de Camino de las Yeseras. Patrimonio Arqueológico de Madrid, 7, Autonomous University of Madrid, Madrid.

Ripollés, E. (2005): Montaña de les Raboses, in Gran Enciclopedia de la Comunidad Valenciana. Levante - El Mercantil Valenciano, Valencia.

Robledo, B., Trancho, G.J. (2003): Análisis antropológico y condiciones de vida de la población argárica de Cerro del Alcázar. Serie Informes Antropológicos. Complutense University, Madrid.

Rojo, M., Garrido, R., Morán, G., Kunst, M. (2005): Del enterramiento colectivo a la tumba individual: el sepulcro monumental de La Sima en Miño de Medinaceli, Soria, España, Boletín del Seminario de Estudios de Arte y Arqueología, LXXI: 11-42.

Romero, H. (1988): La personalidad del Horizonte Necrópolis del Cerro de la Encantada, Cuadernos de Prehistoria y Arqueología de la Universidad Autónoma de Madrid, 11-12 (1): 143-151.

Ruiz Argilés, V., Posac, C. (1956): El Cabezo de La Bastida. Totana (Murcia), Noticiario Arqueológico Hispánico, III/IV: 60-89.

Salanova, L. (2011): Chronologie et facteurs d'évolution des sépultures individuelles Campaniformes dans le nord de la France, Gallia Préhistoire, XLI: 125-142.

Sanahuja, Ma E. (2002): Cuerpos Sexuados. Objetos y Prehistoria. Cátedra, Madrid.

SÁnchez Pravía, J.A. (2001): Proyecto de obras de urbanización del Castillo de Lorca (Fase I), Informe arqueológico inédito, Consejería de Cultura de la Región de Murcia, Murcia.

SÁnchez Romero, M. (2008): Cuerpos de mujeres: la construcción de la identidad y su manifestación durante la Edad del Bronce, Arenal, 15 (1): 5-29.

SÁnchez Romero, M., Alarcón, E. (2012): Lo que los niños nos cuentan: individuos infantiles durante la Edad del Bronce en el sur de la Península Ibérica, in Justel, D. (ed.), Niños en la Antigüedad. Estudios sobre infancia en el Mediterráneo Antiguo. University of Zaragoza, Zaragoza: 57-97.

SCHEUER, L. (2002): Brief Communications: a blind test of mandibular morphology for sexing mandibles in the first years of life, American Journal of Physical Anthropology, 119: 189-191.

Schubart, H. (2004): Das reiche Grab einer jungen Frau aus dem El Argar-zeitlichen Fuente Álamo, Madrider Mitteilungen, 45: 57-78.

Schubart, H. (2012): Die Gräber von Fuente Álamo. Ein Beitrag zu den Grabriten und zur Chronologie der El Argar-Kultur. Madrider Beiträge 32, Deutsches Archäologisches Institut Madrid-Reichert, Wiesbaden.

Schubart, H. Arteaga, O, Pingel, V. (1985): Fuente Álamo. Informe preliminar sobre la excavación de 1985 en el poblado de la Edad del Bronce, Ampurias, 47: 70-107.

Schubart, H., Pingel, V., Arteaga, O. (eds.) (2000): Fuente Álamo. Las excavaciones arqueológicas $1977-$ 1991 en el poblado de la Edad del Bronce. Junta de Andalucía, Sevilla.

Schubart, H., Ulreich, H. (1991): Die Funde der Südostspanischen Bronzezeit aus der Sammlung Siret. Madrider Beiträge, 17, Deutsches Archäologisches Institut Madrid-Philipp von Zabern, Mainz.

SснÜLE, W. (1967): El poblado del Bronce Antiguo en el Cerro de la Virgen de Orce (Granada) y su acequia de regadío, IX Congreso Nacional de Arqueología: 113-121.

SснӥLe, W. (1980): Orce und Galera. Zwei Siedlungen aus dem 3. bis I. Jahrtausend v. Chr. im Südosten der Iberischen Halbinsel. Teil I: Übersicht über die Ausgrabungen 1962-1970. Philipp von Zabern, Mainz. 
Schutkowski, H. (1993): Sex determination of infant and juvenile skeletons: I. Morphognostic features, American Journal of Physical Anthropology, 90: 199-206.

Siret, H., Siret, L. (1890): Las Primeras Edades del Metal en el Sudeste de España. Barcelona.

Soriano, I. (2010): Producción metalúrgica prehistórica en el nordeste de la Península Ibérica (mediados del IV-II milenio cal ANE). Aportaciones cronoculturales, tecnológicas y funcionales. Ph.D. dissertation, Autonomous University of Barcelona.

Strydonck, M. Van, M., Landrie, M., Boudin, M., Grootes, P.M., Nadeau, M.-J., Keppens, E., (2005): Royal Institute for Cultural Heritage Radiocarbon Dates XIX. Brussels [Online Database http://c14.kikirpa.be/]

Turner II, C.G., Nichol, C., Scott, G. (1991): Scoring Procedures for Key Morphological Traits of the Permanent Dentition: The Arizona State University Dental Anthropology System, in Kelly, M., Larsen, C.S. (eds.), Advances in Dental Anthropology. Willey-Liss, New York: 13-31.

Val, E. Del, Sopranis, J.A. Y Posac, F. (1947): Las sepulturas, en Martínez Santa-Olalla, J., Sáez, B., Posac, C., Sopranis, J.A., Val, E. del (1947): Excavaciones en la ciudad del bronce mediterráneo II, de la Bastida de Totana (Murcia). Ministerio de Educación Nacional, Informes y Memorias, 16, Madrid: 91-120.

VAlera, A.C. (2014): Bela Vista 5 - um recinto do final do $3^{\circ}$ milénio a.n.e. (Mombeja, Beja). Era-Arqueologia, 2, Lisboa.

Vlak, D., Roksandic, M., Schillaci, M.A. (2008): Greater sciatic notch as a sex indicador in juveniles, American Journal of Physical Anthropology, 137: 309-315. 